\title{
3 Research Square

\section{Distinct Gut and Vaginal Microbiota Profile in Women with Recurrent Implantation Failure and Unexplained Infertility}

Nayna Patel

Akanksha Hospital and Research Institute

Nidhi Patel

Gujarat Biotechnology Research Centre

Sejal Patel

Gujarat Biotechnology Research Centre

Neelam Nathani

Gujarat Biotechnology Research Centre

Ramesh Pandit

GBRC: Gujarat Biotechnology Research Centre

Molina Patel

Akanksha Hospital and Research Institute

Niket Patel

Akanksha Hospital and Research Institute

Chaitanya Joshi

GBRC: Gujarat Biotechnology Research Centre

Dr Bhavin Parekh ( $\sim$ bp2580@gmail.com )

Akanksha Hospital https://orcid.org/0000-0001-6237-4727

\section{Research}

Keywords: Gut microbiota, Vaginal microbiota, Implantation failure, Infertility, Endometrium

Posted Date: July 14th, 2021

DOl: https://doi.org/10.21203/rs.3.rs-672281/v1

License: (c) (1) This work is licensed under a Creative Commons Attribution 4.0 International License.

Read Full License

Version of Record: A version of this preprint was published at BMC Women's Health on April 12th, 2022. See the published version at https://doi.org/10.1186/s12905-022-01681-6. 

1 Distinct gut and vaginal microbiota profile in women with Recurrent

2 Implantation Failure and Unexplained Infertility

3 Author details

4

5 Nayna Patel ${ }^{1}$, Nidhi Patel ${ }^{2}$, Sejal Pal ${ }^{1}$, Neelam Nathani ${ }^{2}$, Ramesh Pandit ${ }^{2}$, Molina

6 Patel $^{1}$, Niket Patel ${ }^{1}$, Chaitanya Joshi ${ }^{2}$ and Bhavin Parekh ${ }^{1 *}$

7 1. Akanksha Hospital and Research Institute, Lambhvel Road, Lambhvel, Anand, 8 Gujarat 387310, India.

9 2. Gujarat Biotechnology Research Centre (GBRC), Gandhinagar, Gujarat, 10 382011, India.

12 Author's information

13 E-mail addresses of authors:

14 *Correspondence: Dr Bhavin Parekh: bp2580@gmail.com

15 Nayna Patel: nayana@ivf-surrogate.com

16 Nidhi Patel: patelnidhi702@gmail.com

17 Sejal Pal: sejalpal15@gmail.com

$18 \quad$ Neelam Nathani : neelam.nathani13@gmail.com

19 Ramesh Pandit : panditrameshj@gmail.com

20 Molina Patel: Drmolinapatel@gmail.com

21 Niket Patel: niketslam@gmail.com, Dr Chaitanya Joshi: director.gbrc@gmail.com 


\section{Abbreviations}

24 AMH, Anti-Müllerian hormone; ASV, Amplicon sequence variants; BMI, Body 25 mass index; ET, Embryo transfer; (F/B) ratio, Firmicutes to Bacteroidetes ratio; 26 ICSI, Intracytoplasmic Sperm Injection; IL-6, Interleukin 6, IL-10, Interleukin 10, 27 IVF, In vitro fertilization; L. delbrueckii, Lactobacillus delbrueckii; L. 28 equicursoris, Lactobacillus equicursoris; L. fermentum, Lactobacillus fermentum;

29 L. gasseri, Lactobacillus gasseri; L. Iners, Lactobacillus iners; L. jensenii, 30 Lactobacillus jensenii; L. reuteri, Lactobacillus reuteri; L. ruminis, Lactobacillus

31 ruminis; L. salivarius; Lactobacillus salivarius; L. vaginalis; Lactobacillus 32 vaginalis; LDA, Linear discriminant analysis; LEfSe, linear discriminant analysis 33 effect size; LPS, lipopolysaccharides; MAMPs, Microbe-associated molecular 34 patterns; OTU, operational taxonomic units; PERMANOVA, Permutation 35 multivariate analysis of variance; PCoA, Principle Coordinate Analysis; RIF, 36 Recurrent implantation failure; SCFA, short-chain fatty acids; 16S rna, 16S 37 ribosomal RNA; TLRs, toll-like receptors, TMAO Trimethylamine N-oxide; TNF$38 \alpha$, Tumor Necrosis Factor- $\alpha$; UE, Unexplained infertility. 
42 Background: Implantation failure limits the success rate of natural and in 43 vitro fertilization (IVF)-assisted conceptions. Evidence suggests dysbiosis in the 44 female reproductive tract impacts implantation failure. However, whether gut 45 dysbiosis influences implantation failure and whether it accompanies reproductive 46 tract dysbiosis remains unexplored.

47

48 Method: We recruited 11fertile women as the controls, and a cohort of 20 women 49 diagnosed with implantation-failure associated infertility, which included 10 50 women diagnosed with recurrent implantation failure (RIF), and 10 women 51 diagnosed with unexplained infertility (UE). Using next-generation amplicon 52 sequencing, we compared the diversity, structure, and composition of fecal and 53 vaginal bacteria of the controls with that of the infertile cohort. While we 54 sequenced fecal samples of all the participants $(n=31)$, we could only 55 sequence 8 vaginal samples in each group $(n=24)$.

57 Results: Compared with the controls, $\alpha$-diversity of the gut bacteria, analysed by 58 Chao 1 and Shannon indices, among the infertile groups declined $(p<0.05)$. $\beta$ 59 diversity between the controls and infertile cohort, measured by both Bray-Curtis 60 and Jaccard distances, differed significantly $(p<0.05)$. Taxa analysis of the gut 61 bacteria revealed enrichment of Gram-positive bacteria, mainly of the 
62 phylum Firmicutes, in the RIF group. In contrast, Gram-negative bacteria were 63 relatively more abundant in the UE group. Additionally, mucus-producing 64 bacteria genera such as Prevotella and Sutterella declined in the infertile 65 cohort $(p<0.05)$. Intriguingly, significant enrichment $(p<0.05)$ of the 66 genus Hungatella, associated with trimethylamine N-oxide (TMAO) production, 67 occurred in the infertile cohort. Vaginal microbiota was dominated by $L$. 68 iners across the groups, with the UE group showing the highest levels. Of the three 69 groups, the RIF group had the least diverse vaginal microbiota. Taxa analysis 70 showed higher levels of anaerobic bacteria such 71 as Leptotrichia, Snethia, and Prevotella in the controls.

Conclusion: We posit that in the setting of the compromised gut mucosal barrier,

74 the phyla Firmicutes generates TNF- $\alpha$-driven systemic inflammation, leading to 75 RIF, whereas an overload of Gram-negative bacteria induces IL-6-driven systemic 76 inflammation, leading to UE. Additionally, Hungatella-induced elevation 77 of TMAO levels causes platelet hypercoagulability, synergistically contributing to 78

81 Key Words: Gut microbiota, Vaginal microbiota, Implantation failure, 82 Infertility, Endometrium 


\section{Introduction}

86 Infertility, defined as a failure to conceive after 1 year of appropriately timed 87 unprotected intercourse, is a distressing and costly reproductive disorder that 88 affects up to $15 \%$ of couples globally (Kamel 2010). Some known causes of 89 infertility include pelvic diseases, peritoneal factors, cervical factors, ovulatory 90 disorders, reproductive aging of women, and male factors (Lindsay and Vitrikas 91 2015).

92 Despite this knowledge, some couples are diagnosed as having unexplained 93 infertility (UE) because the underlying mechanism(s) remain undefined even after 94 assessment of ovulatory function, tubal patency, and sperm parameters (Bellver, 95 Soares et al. 2008). Frustratingly, many of the infertile couples undergo multiple 96 unsuccessful assisted reproduction technology (ART) cycles (i.e., IVF and/or 97 intracytoplasmic sperm injection (ICSI)) and are thus diagnosed as having repeated 98 implantation failure (RIF) (Bellver, Soares et al. 2008, Simon and Laufer 2012, 99 Polanski, Baumgarten et al. 2014). Yet another subgroup of infertile couples, 100 diagnosed as recurrent pregnancy loss (RPL), exists that conceive several times 101 ( $\geq 3)$, but miscarriage occurs each time before gestational week 28, although 102 controversies exist on its definition (Christiansen, Nielsen et al. 2006, El Hachem, 103 Crepaux et al. 2017). It has been argued that RIF and RPL represent the same 104 condition spectrum (Christiansen, Nielsen et al. 2006). 
105 Pathologies of all these enigmatic conditions converge on mechanisms by which 106 the embryo fails to implant in the uterus-also called implantation failure 107 (Graham, Seif et al. 1990, Christiansen, Nielsen et al. 2006, Bellver, Soares et al. 108 2008, Simon and Laufer 2012). It is the main limiting factor for natural and in vitro 109 fertilization (IVF)-assisted pregnancies (Bellver, Soares et al. 2008, Hernández110 Vargas, Muñoz et al. 2020). Failed implantation involves a triumvirate of a poor 111 quality embryo and an unreceptive endometrium and ill-timed embryo112 endometrium interaction, of which unreceptive endometrium appears to be the 113 most critical factor. (Simon and Laufer 2012). In fact, unreceptive endometrium 114 has been implicated in two-thirds of all the failures (Simon and Laufer 2012). 115 While multiple factors that disrupt endometrium receptivity, including steroidal 116 hormonal imbalance, thrombotic abnormalities, hyperhomocysteinemia, and 117 immune dysfunctions, have been identified, much remains recondite (Bellver, 118 Soares et al. 2008, Simon and Laufer 2012, Cho, Kim et al. 2019).

119 The reproductive tract bacteria influence implantation failure of endometrial origin 120 (Al-Nasiry, Ambrosino et al. 2020). For example, women with non-Lactobacillus121 dominated microbiota in a receptive endometrium had decreased implantation rates 122 than women with a Lactobacillus-dominated (>90\%) microbiota (Moreno, 123 Codoñer et al. 2016). Chlamydia species in the endocervix of women undergoing 124 IVF-ET were correlated with implantation failure (Witkin, Sultan et al. 1994). 
125 Increasingly researchers are exploring how the local microbiota influences 126 physiology at distal sites, especially how the gut bacteria, the densest and most 127 diverse bacterial communities of the body, impact distal organs, including the brain 128 and lungs, leading to the notions of the gut-brain axis and gut-lung microbiota axis 129 (Ravel and Brotman 2016, Budden, Gellatly et al. 2017). However, hitherto, gut 130 bacteria's role in implantation failure of endometrial origin remains unexplored, let 131 alone the gut-reproductive tract microbiota axis, even though a compelling 132 rationale exists. Indeed, the gut bacteria impact the immune system, hormonal 133 homeostasis, and the coagulation system - all of which mediate implantation 134 success (Alexander, Targan et al. 2014, Baker, Al-Nakkash et al. 2017, Vinchi 135 2019). The comorbidity of gut disorders (e.g., celiac disease) with infertility 136 disorders, including recurrent pregnancy loss, allude to a role of 'gut-reproductive 137 tract axis' in implantation failure (Tersigni, D'Ippolito et al. 2018).

138 Hence we investigated whether gut dysbiosis occurs in women with implantation 139 failure, and if so, whether it accompanies vaginal dysbiosis, which usually involves 140 a decline in either the levels of Lactobacillus or protective species of Lactobacillus 141 and a simultaneous rise in the diversity and density of other bacteria (Madhivanan, 142 Alleyn et al. 2015, Amabebe and Anumba 2018, Wang, Fan et al. 2020). To this 143 end, by using 16S rRNA gene sequencing, we compared the diversity, structure 
144 and taxonomic composition of the fecal and vaginal microbiota of fertile women 145 with that of infertile women with a history of RIF and UE.

146 


\section{Materials and Methods}

$148 \quad 2.1$ Study participants

149 For this retrospective, single-center cohort study, fertile and infertile women, 150 referred to Akanksha Hospital and Research Institute between September 2018 and

151 February 2019, were recruited and divided into three groups: the control, RIF, and 152 UE groups. The RIF group's inclusion criteria were women who could not 153 conceive after $\geq 2$ fresh IVF-embryo transfer cycles/ Intracytoplasmic Sperm 154 Injection (ICSI) or had $\geq 3$ consecutive miscarriages. UE was diagnosed if a cause 155 remains undefined after our routine fertility tests with the following criteria: 156 infertility of more than 1 year, normal male partner, normal menstrual rhythm with 157 regular ovulation, and normal hormonal tests (i.e., thyroid, prolactin, AMH) 158 Exclusion criteria included diabetes, polycystic ovary syndrome and 159 endometriosis, diarrhea, ongoing pregnancy, addiction (e.g., drugs, alcohol, 160 tobacco etc.) and the use of antibiotics within at least two weeks before sample 161 collection.

\section{$162 \quad 2.2$ Ethical approval and Consent to participate}

163 We performed all the sampling and experiments with the approval of the local 164 Ethics Committee of Sat Kaival Hospital Pvt. Ltd (EC2013/053). Participants gave 165 their oral and written informed consent for fecal and vaginal sample collections 
166 and subsequent microbiological analysis. We recorded and compared participants' 167 characteristics (Table 1).

\section{$168 \quad$ 2.3 Sample Collection}

169 The fecal and vaginal samples were freshly and simultaneously collected. 170 Participants collected the fecal samples in a sterile plastic container with a tight 171 closing lid. To collect the vaginal samples, using a sterile swab stick, clinicians 172 thoroughly wiped the posterior fornix of the vagina of the participants. These 173 swabs were stored in sterile vials. Both types of samples were packaged and first 174 placed in a frozen storage at $-20^{\circ} \mathrm{C}$ in the hospital and later, within 24 hours, 175 transported on ice to be stored at $-80^{\circ} \mathrm{C}$ at Gujarat Biotechnology Research Centre 176 (GBRC) for further analysis.

\subsection{DNA extraction}

179 DNA extraction was performed from approximately $200 \mathrm{mg}$ of fecal samples and $180 \sim 1 \mathrm{ml}$ of thoroughly vortexed swab sample using QIAamp DNA Stool Mini Kit 181 according to the manufacturer's instructions. Total DNA was eluted in $30 \mu \mathrm{L}$ of 182 AE buffer. DNA concentration was quantified fluorometrically with a Qubit 2.0 183 dsDNA HS Assay kit. DNA was stored at $-20^{\circ} \mathrm{C}$ for further procedures. 


\subsection{Library preparation and 16s rRNA Sequencing}

187 The V2-V3 hypervariable regions of the 16S rRNA gene were amplified using 188 fusion primers, 101 F5'ACTGGCGGACGGGTGAGTAA $\quad 3^{\prime}$ and $518 \mathrm{R}$ 189 5'CGTATTACCGCGGCTGCTGG 3'. Amplicon libraries were purified using the 190 Agencourt AMPure XP (Beckman Coulter). For quality control, we used 191 Bioanalyzer with a DNA-HS assay kit. All the libraries were quantified using 192 Qubit fluorimeter v4.0 and were pooled into equimolar concentrations. Clonal 193 amplification (Emulsion PCR) sequencing was performed on the Ion S5 system 194 using the Ion 520/530 kit OT2 (ThermoFisher Scientific) with Ion One Touch 2 195 system, which uses a 530 chip on Ion S5 plus sequencer according to the 196 manufacturer's instructions.

\section{$197 \quad 2.6$ Bioinformatics and Statistical analysis}

\section{Diversity}

199 Microbial community richness and diversity was evaluated by $\alpha$-diversity that 200 included Chao1 and Shannon indices. The Kruskal-Wallis test used to determine 201 statistical differences between the three groups. The Mann-Whitney U test was 202 used to determine the influence of diet on $\alpha$-diversity. $\beta$-diversity, differences in 203 microbial community structures between the three groups, was analysed using 204 Principle Coordinate Analysis (PCoA) based on Bray-Curtis and Jaccard distances, 
205 and the statistical difference between the groups was calculated using non206 parametric permutational multivariate analysis of variance (PERMANOVA).

207 QIIME2 software was used to calculate alpha and beta diversity indices. The 208 demultiplexed sequences were uploaded to QIIME2 environment, and denoising 209 was carried out using DADA2. Amplicon sequence variants (ASVs) were 210 predicted at a minimum sampling depth of 25000 for Gut datasets, and 9000 for the

211 vaginal datasets. The predicted ASVs were taxonomically classified using the pre212 trained classifier of the full $16 \mathrm{~S}$ rRNA gene sequence of the SILVA database.

\section{Taxonomic structure}

214 We analysed the gut microbiota at the community level to determine differences in 215 the microbial composition between groups and to identify taxa with significantly 216 different abundance (relative abundance $>0.001$ and $\mathrm{P}<0.05$ ). Additionally, linear 217 discriminant analysis effect size (LEfSe) method was employed to identify species 218 with significant differences in abundance between the three groups ( $|\mathrm{LDA}|>3$ and $219 \mathrm{P}<0.05)$. Kruskal-Wallis and Mann-Whitney $\mathrm{U}$ tests were performed 220 for computing statistical differences between the groups at taxonomic levels in the 221 study viz., three major groups and the infertile cohort versus controls using 222 STAMP v2.1 software (Parks et al., 2014). 
223 Continuous data are presented as mean \pm standard deviation (SD) or frequencies

224 (number and percentages), calculated using GraphPad Prism statistical software

225 6.0. To determine statistical differences in subjects' characteristics between 226 groups, we performed one-way ANOVA followed by post-hoc Tukey testing.

227 


\section{Results}

\subsection{Clinical Characteristics among the Control, RIF, and UE Groups}

230 We included 31 study subjects, comprising 11 fertile women, enrolled as the 231 control group, 10 women with a history of RIF, and 10 women with a history of

232 UE (Table 1). Comparisons of the mean age between the three groups showed that 233 the controls were statistically significantly younger than the RIF group ( $F=6.8, p$ $234<0.01$ ), while the UE group was older than the controls and younger than the RIF 235 group, although both differences were statistically insignificant (Table 1). The 236 average BMI was not significantly different between the controls and the UE group 237 and between the RIF and UE groups but was significant between the controls and 238 the RIF group $(\mathrm{F}=3.7, \mathrm{p}<0.05)$ (Table 1). Among women with RIF, eight were 239 nullipara (80\%), and two were nulligravida (20\%), while all women with UE $240(100 \%)$ were nullipara. There were more vegetarian participants in the UE group (9 241 of $10 ; 90 \%)$ than in the RIF group (4 of $10 ; 40 \%)$ and the controls (4 of $11 ; 36 \%)$ 242 (Table 1).

\subsection{Metagenomics Findings of Gut and Vaginal bacteria}

245 In total 31 fecal samples and 24 vaginal swab samples, sequencing of the V2-V3 246 region of the $16 \mathrm{~S}$ rRNA gene created 235810 sequences, with an average of 1,01 , 247767 sequences, and 178260 high-quality sequences, with an average of 3,76,012 
248 sequences, per sample for fecal and vaginal samples respectively. Based on the 249 results of the operational taxonomic units (OTUs) analysis, rarefaction curves 250 show that the sequencing depth was adequate to analyse the gut and vaginal 251 bacteria in the three groups (Figures S1 (a), (b) and Figures S2 (a), (b)).

\subsection{Richness and diversity of Gut bacteria}

254 We performed bacterial diversity analyses by comparing the richness using Chao 1 255 and evenness using the Shannon index for the fecal microbial communities 256 between the three groups. We found that richness differed significantly between 257 the three groups (Chao 1 index (Kruskal-Wallis test, $p=0.049)$ ) (Figure 1(a)). 258 Precisely, the controls had a significantly higher richness than the RIF group 259 (pChao $1=0.04$ ) and UE group (pChao $1=0.03$ ). Richness was similar between 260 RIF and UE groups (pChao $1=0.75$ ). We discovered that evenness differed 261 significantly between the three groups: Shannon index (Kruskal-Wallis test, $p=$ 262 0.003) (Figure 1(b)). Specifically, the controls had more evenness than the RIF 263 (pShannon $=0.006)$ and the UE groups $($ pShannon $=0.002)$. In contrast, evenness 264 was similar between the RIF and the UE groups (pShannon =0.65). Interestingly, 265 the diet did not affect alpha diversity $(\mathrm{pShannon}=0.165)($ Figure 3S).

266 Regarding the bacterial community structure differences, the PCoA plot of the 267 Bray-Curtis and Jaccard dissimilarity showed that bacteria of the RIF and UE 
268 groups overlapped and that both groups differed markedly from the controls

269 (Figures 2(a), (b)). In the PCoA plot based on Bray-Curtis distances, the first and 270 second axes of the PCoA explained $21.5 \%$ of the total variance with a significant 271 difference (PERMANOVA, P $<0.05, \mathrm{R} 2=0.12$; Figures 2(a)). Showing the similar 272 clustering pattern, in the Jaccard based PCoA plot, the first and second axes 273 explained $23.4 \%$ of the total variance with a significant difference 274 (PERMANOVA, P<0.05, R2 =0.10; Figures 2(b)).

275

$276 \quad 3.4$ Taxonomic analysis of Gut bacteria

277 After excluding the sequences that were present in less than $3 \%$, we clustered the 278 high-quality sequences into OTUs and assigned taxonomic identities. 279 Consequently, we found 550 OTUs pertaining to 481 genera, 265 families, 156 280 orders, 68 classes, 715 species and 28 phyla. To evaluate the contribution of 281 different taxa to diversity and composition, we calculated the relative abundance of 282 taxa at the phylum, family and genus levels. Except at the genus level, we could 283 not find statistically significant alteration of particular taxa at any other levels. 284 Though, noticeable trends from a clinical point of view were still apparent in the 285 data. 
286 Across all 31 participants, the phyla level composition, represented by read 287 percentages, showed usual human microbiota structures. Overall, among the 288 detected 28 phyla, the four most abundant microbes were Firmicutes $(85.10 \%)$, 289 Bacteroidetes (7.70\%), Proteobacteria (4.75\%), and Actinobacteria (1.8\%) 290 (Figure 3(a)). With a relative abundance of less than 1\%, the remaining bacterial 291 population belonged to four phyla, including Verrucomicrobia, Tenericutes, 292 Cyanobacteria, and Chloroflexi.

293 Firmicutes were abundant (7-9 \%) in the RIF group than both the control and UE 294 groups. Firmicutes were only slightly more abundant $(2 \%)$ in the UE group than 295 the controls. Bacteroidetes were depleted (50\% less) in the RIF group than the 296 other two groups. In contrast, the control and UE groups had the same levels of 297 Bacteroidetes. Proteobacteria were relatively less abundant (4-7\% less) in the RIF 298 group than the control and UE groups (Figure 3 (a)). Among the three groups, the 299 UE group had the highest levels of Proteobacteria, almost 2 fold higher than the 300 controls. Actinobacteria were depleted in the UE group ( 4 fold less) compared to 301 the other two groups, with the RIF group showing the highest abundance amongst 302 all the groups, with 2 fold more abundance than the control group (Figure 3(a)).

303 The dominant bacterial families for all the subjects in the descending order of 304 abundance were Lachnospiraceae, Ruminococcaceae, Bifidobacteriaceae, 
305 Erysipelotrichaceae, Lactobacillaceae, Prevotellaacae, Vellinollacaea and 306 Enterobacteriaceae (Figure 3(b)). The levels of Lachnospiraceae and 307 Ruminococcaceae were similar between the three groups. Notably, the levels of 308 Lactobacillaceae and Prevotellaacae families were highest in the controls as 309 compared to the other two infertile groups. Bacteroidaceae, Vellinollacaea and 310 Enterobacteriaceae were highest in the UE group compared to the RIF and control 311 groups, while Bifidobacteriaceae and Erysipelotrichaceae families were highest in 312 the RIF group as compared to the control and UE groups (Figure 3(b)).

313 We further determined statistical differences in the specific bacterial genera of the 314 three groups. Among 481 genera, the 6 were statistically significantly $(p<0.05)$ 315 different: Bacteroides, Prevotella 9, Hungatella, Ruminococcaceae UCG-004, 316 Ruminococcaceae UCG-010, and Sutterella. Aside from Bacteroides and 317 Prevotella 9, the abundance of the other 5 genera, while statistically significant $318(\mathrm{p}<0.05)$, occurred in much lower proportions $(<1 \%)$. Notably, Bacteroides and 319 Hungatella were abundant in the infertile cohort, especially in the UE group, than 320 the controls (Figure 3(c)).

321 Bacteroides and Prevotella 9, the two most highly abundant genera, varied 322 significantly between the three groups. Compared to the controls, the relative 323 abundance of Prevotella 9 plunged noticeably in the RIF group (7 fold), and the 
324 UE group (8 fold), and the relative abundance of Bacteroides rose in the RIF group 325 (1.5 fold) and UE group (3.2 fold) (Figure 3(c)). When we compared the infertile 326 group against the controls, we found that in the infertile cohort Prevotella 9, 327 Ruminococcaceae UCG-004, Ruminococcaceae UCG-010 (p<0.05) declined, 328 whereas Bacteroides, Dorea, oral clone FR58 and Peptoniphilus increased $329(\mathrm{p}<0.05)$ (Figure 4S).

\subsection{LEfSe analysis}

LEfSe analysis was performed to assess the differentially abundant communities in 332 the three groups (Figure 4 (a), (b)). In the controls, we observed diverse microbial 333 communities with a high LDA score $(\log 10)$, with Clostridia showing the highest 334 LDA score $>9(\mathrm{p}<0.05)$. In the RIF group, only Eubacterium halli showed the 335 highest dominance with an LDA score $>7$ ( $<0.05)$. In the UE group, 336 unconventional Firmicutes such as Veillonellaceae, Selenomonadales, of the class 337 Negativicutes, showed the highest preponderance with an LDA score $>7(\mathrm{p}<0.05)$.

\subsection{Metagenomics of vaginal bacteria}

339 Across the 24 vaginal samples, we found 384 distinct species belonging to 301 340 different genera classified in 135 different families, distributed into 10 phyla. We 341 compared taxa between the three groups. Given the small sample size, we could 
342 not detect a significant statistical difference between them. Nonetheless, interesting

343 trends in the data were apparent from a clinical point of view.

\section{$344 \quad 3.7$ Taxonomic Analysis of Vaginal Bacteria}

345 In the descending order, the dominant phyla, among the 10 detected phyla, 346 included Firmicutes, Fusobacteria, Proteobacteria, Actinobacteria, Bacteroidetes, 347 and Patescibacteria (Figure 5(a)). Of these, Firmicutes accounted for the vast 348 majority of the vaginal bacteria in all the groups, with both the RIF (69\%) and UE 349 $(69.71 \%)$ groups showing similar relative abundance, which was higher than the 350 controls (53\%). Fusobacteria (18\% Vs. 0.07 Vs. 0.14) and Bacteriodetes $(4.1 \%$ 351 Vs0.17 Vs. 0.92) were relatively abundant in the controls than the RIF and UE 352 groups. Proteobacteria were marginally more abundant in both RIF (15\%Vs.11\%) 353 and UE (19\% Vs. 11\%) groups compared to the controls (Figure 5(a)).

354 The dominant families for all the groups in the descending order of abundance 355 were Lactobacillaceae, Bifidobacteriaceae, Leptotrichiaceae, and Prevotellaceae

356 (Figure 5(b)). Lactobacillaceae and Bifidobacteriaceae were present in all the 357 groups. Reflecting this trend at the phylum level, levels of Lactobacillaceae were 358 higher in all the groups, with UE (65.3\%) and RIF (58.41\%) women showing the 359 highest levels compared with the controls (47.2\%). 
360 At the genus level, 5 genera were detected, of which three were present in all the 361 groups (Figure 5(c)). Lactobacillus was the most dominant genus among them,

362 followed by Gardnerella and Parvimonas. Gardnerella, Prevotella, Parvimonas, 363 and Snaethia were relatively more abundant in the controls compared to the 364 infertile groups. Compared to the controls, Lactobacillus was relatively more 365 abundant in the RIF and the UE groups (2-fold).

366 At the species levels, Sneathia ammni (0.36\%) was detected only in the control 367 groups. In contrast, Lactobacillus iners $A B$ - 1 werepresent in all the groups, with 368 descending order of relatively high abundance in the following manner: the UE 369 group (62\%), the controls (16\%), and the RIF group (11.02\%) (Figure 5(d)).

$370 \quad 3.8$ LEfSe analysis

371 We performed LEfSe analysis to assess the differentially abundant vaginal 372 bacterial communities in the three groups. We could only find significant 373 differences in the controls, with Leptotrichia, of the phylum Fusobacteria, 374 showing an LDA $(\log 10)$ score $>3(\mathrm{p}<0.05)$ (Figure 6).

$375 \quad 3.9$ Alterations of the genus Lactobacillus Species

376 Within the genus of Lactobacillus, 9 species were identified. L. gasseri, L. ruminis, 377 and L. iners $A B-1$ were found in all the groups, with Lactobacillus iners $A B-1$ 378 being the most abundant species (Figure 7). Among these, L. jensenii and $L$. 
379 vaginalis were only detected in the UE group, while L.reuteri was unique to the

380 RIF group. L. equicursoris, L. fermentum and L. salivarius were unique to the 381 controls.

382 


\section{Discussion}

384 We compared, for the first time, the gut-vaginal microbiota axis of fertile women 385 with that of women diagnosed with RIF and UE. The core findings include i) the 386 infertile groups had gut dysbiosis as evident by low $\alpha$-diversity indices and beta 387 diversity metrics; ii) the gut microbial composition of the RIF and UE groups 388 differed noticeably, with a set of Gram-positive taxa, mainly members of the 389 phylum Firmicutes, being dominant in the former group and a set of Gram390 negative bacteria, comprising members of the phylum Proteobacteria and the class

391 Negativicutes, of the phylum Firmicutes, being dominant in the latter group; iii) 392 butyrate-producing genera such as Prevotella declined in the infertile cohort; iv) 393 elevated levels of the genus Hungatella occurred in the infertile cohort, especially 394 in the UE; and v) the infertile cohort, especially the RIF group, had a 395 comparatively healthy vaginal microbiota than the controls.

396 Gut microbial richness and diversity, defined by $\alpha$-diversity indices, declined 397 amongst the infertile groups, with the highest decline in the UE group. Studies 398 have suggested that reduced $\alpha$-diversity, an important indicator of gut microbiome 399 health, indicates low-grade inflammatory disorders such as inflammatory bowel 400 disease, metabolic disorder and obesity (Ott and Schreiber 2006, Le Chatelier, 401 Nielsen et al. 2013, Al-Assal, Martinez et al. 2018). Of note, diet (vegetarian vs. 402 Non-vegetarian) had little influence on $\alpha$-diversity. Although, a fine-grained 
403 analysis showed vegetarian women had a higher proportion of the phyla 404 Patescibacteria and the genus Bacteroides, likely reflecting its role in the digestion 405 of plant fiber (Matijašić, Obermajer et al. 2014, Jang, Choi et al. 2017). Gut 406 dysbiosis was also reflected in beta diversity indices, suggesting a distinct bacterial 407 composition between the infertile cohort and the controls.

408 Notably, the reduction in the richness of gut bacteria in the RIF group was seen for 409 the phyla Bacteroidetes and Proteobacteria compared with the other groups. 410 Mirroring this, we observed a lower percentage of prevotellaceae (phylum 411 Bacteroidetes), Veillonellaceae (phylum Proteobacteria), Enterobacteriaceae 412 (phylum Proteobacteria). This was partly reflected at the genus level by a several413 fold decrease in the genus Prevotella (phylum Bacteroidetes) and a mild elevation 414 in Bacteroidaceae (phylum Bacteroidetes). In contrast, in the UE group, the 415 reduction in richness was seen mainly for the phyla Actinobacteria and marginally 416 for Bacteroidetes. In keeping with this, reduced levels of the Bifidobacteriaceae 417 family (Phylum Actinobacteria) were noted, and a marginal decline in the phyla 418 Bacteroidetes was reflected by the depletion of the prevotellaceae family (phylum 419 Bacteroidetes) and a modest rise in Bacteroidaceae (phylum Bacteroidetes).

420 Altogether, the data clearly indicate that the reduced microbial richness in the RIF 421 and UE groups is characterised by contrasting types of abundance at the taxa level. 422 Nonetheless, data showed a partial overlap at the genus level in that a relative 
423 decline in the abundance of Prevotella and an increase in the abundance of 424 Bacteroides commonly occurred in both the groups. Since Prevotella builds the 425 protective gut mucosal barrier from short-chain fatty acids (SCFAs) such as 426 butyrate and Bacteroides impedes mucin synthesis by producing metabolites such 427 as succinate, acetate, and propionate, these findings suggest thinned mucosal 428 protection is the common abnormality in the infertile cohort. (Mejía-León and 429 Barca 2015). Of note, although not highly abundant in the infertile cohort, 430 Ruminococcaceae UCG-004 and Ruminococcaceae UCG-010, of the 431 Ruminococcaceae family and the genus Sutterella declined as well. These are also 432 butyrate- producing genera (Vital, Karch et al. 2017, Jennings, Koch et al. 2019, 433 Wang, Wichienchot et al. 2019).

434 When the mucus barrier is thinned, the gut bacteria and other microbe-associated 435 molecular patterns (MAMPs) come in direct contact with toll-like receptors 436 (TLRs), located in the gut epithelial cells such as the Paneth cells (Uchida, 437 Oyanagi et al. 2014, Okumura and Takeda 2017, Steimle, Michaelis et al. 2019). 438 TLRs recognise microbes and MAMPs, and subsequently elicit an immune 439 response, leading to localised and systemic inflammation, which can cause 440 implantation failure (Uchida, Oyanagi et al. 2014, Mejía-León and Barca 2015, 441 Okumura and Takeda 2017, Steimle, Michaelis et al. 2019). 
442 We proffer the following hypotheses to explain how the gut dysbiosis in the 443 infertile groups causes implantation failure by separate mechanisms that promote 444 systemic inflammation. We posit that gut dysbiosis-induced metabolic 445 dysregulation plays a role in RIF. At the phyla level, abundances of Bacteroidetes 446 and Proteobacteria were lower. By contrast, Firmicutes and Actinobacteria's 447 levels were higher in the RIF group compared with the other two groups-448 indicating an obesity-associated microbiota profile (Koliada, Syzenko et al. 2017). 449 Obesity has been linked to an increase in Firmicutes to Bacteroidetes (F/B) ratio 450 (Verdam, Fuentes et al. 2013, Koliada, Syzenko et al. 2017).

451 Conversely, weight loss has been shown to normalise this ratio (Koliada, Syzenko 452 et al. 2017). The F/B ratio was elevated in six out of ten women in the RIF group. 453 In contrast, only two to three subjects had elevated F/B ratio in the other groups. 454 Unsurprisingly, the RIF group's mean BMI, highest among the three groups, was 455 in the obesity range (obesity $\geq 25 \mathrm{~kg} / \mathrm{m}^{2}$ for Asian Indians (Mahajan and Batra 456 2018)), which chimes with the fact that obesity is a risk factor for RIF(Zhang, Liu 457 et al. 2019).

458 Notably, the Clostridium XIVa cluster, of the Firmicutes phylum, whose members 459 comprise flagellated bacteria with a tendency to colonise mucus, play a critical role 460 in metabolic dysregulation such as obesity (including visceral 461 adiposity) (Lopetuso, Scaldaferri et al. 2013, Verdam, Fuentes et al. 2013, 
462 Jennings, Koch et al. 2019). Indeed, a trend towards an increase in the relative 463 abundance of Firmicutes genera in this cluster such as Lachnoclostridium, Dorea,

464 Ruminococcus 2, and Eubacterium was duly noted in the RIF group (Lopetuso, 465 Scaldaferri et al. 2013). Crucially, LEfSe analysis found that Eubacteriumhalli, a 466 member of this cluster, previously found to be elevated in human obesity, is a RIF 467 biomarker (Sanz, Santacruz et al. 2008, Verdam, Fuentes et al. 2013). Strikingly, 468 the RIF group had the highest levels of the Erysipelotrichaceae (phylum 469 Firmicutes) family. In contrast, it was almost absent in the two groups. Elevated 470 levels of Erysipelotrichaceae has been linked to human obesity and has been 471 correlated to elevated levels of Tumor Necrosis Factor-alpha (TNF- $\alpha$ ), a pro472 inflammatory cytokine involved in obesity-linked insulin resistance (Kaakoush 473 2015). Tellingly, a high relative abundance of Firmicutes has been shown to 474 correlate with increased levels of peripheral TNF- $\alpha$ (Orbe-Orihuela, Lagunas475 Mart $\tilde{A}-n e z$ et al. 2018). A rodent study found that a high-fat diet first increased the 476 phylum Firmicutes, corresponding with the changes of Paneth cell-antimicrobial 477 peptides, which was later followed by the elevations of circulating inflammatory 478 cytokines, including TNF- $\alpha$, thus establishing causality between the phylum 479 Firmicutes and TNF- $\alpha$ (Guo, Li et al. 2017).

480 Therefore, precisely, we postulate that the phylum Firmicutes generates TNF- $\alpha$ 481 driven systemic inflammation, and consequent insulin resistance may cause RIF. 
482 Strikingly, investigators showed elevated TNF- $\alpha / \mathrm{IL}-10$ ratio correlates with an 483 increased risk of IVF failure (Winger, Reed et al. 2011). Chan et al. found that 484 insulin resistance reduces implantation rate in in vitro maturation-in vitro 485 fertilization-embryo transfer cycle (Chang, Han et al. 2013). Metformin, known to 486 reduce the F/B ratio, has been shown to increase pregnancy rate in IVF repeaters 487 without polycystic ovary syndrome (Jinno, Watanabe et al. 2010, Wang, Saha et al. 488 2018). Investigator showed an arginine-rich diet, known to reduce obesity and 489 increase insulin sensitivity, corrects the elevated F/B ratio and increases embryo 490 survival (Dai, Wu et al. 2011).

491 The most striking phyla level changed in the UE group involved depletion of 492 Actinobacteria and abundance of Proteobacteria, the pro-inflammatory phylum, 493 comprising common pathogens (e.g., Escherichia, Salmonella) (Sekirov, Russell et 494 al. 2010). Compared to the controls, only marginal changes occurred in the levels 495 of phyla Firmicutes and Bacteroidetes in this group, suggesting a critical role of 496 Proteobacteria phyla in UE. This concurs with the fact that Bifidobacterium, a 497 genus of the depleted phyla Actinobacteria, inhibits gut pathogens (Azad, Sarker et 498 al. 2018). Unsurprisingly, compared to the other two groups, the UE group had the 499 highest enrichment of pathogenic Gram-negative families, whose outer membrane 500 contains a unique component, lipopolysaccharide (LPS) (Brown 2019). These 501 bacterial families included: Bacteroidaceae (phylum Bacteroidetes), 
502 Veillonellaceae (phylum Firmicutes) and Enterobacteriaceae (phylum

503 Bacteroidetes). Cogently, LEfSe analysis revealed members of the Negativicutes

504 class-such as Veillonellaceae, Selenomonadales, which are atypical gram505 negative Firmicutes, which possess lipopolysaccharides in the outer membranes506 were biomarkers of UE (Vesth, Ozen et al. 2013).

507 The abundance of Negativicutes has been linked with an increase in the systemic 508 levels of IL-6, the pro-inflammatory cytokine (Leite, Rodrigues et al. 2017). Along 509 this line, enrichment of other Gram-negative species has been shown to increase 510 plasma IL-6 levels (de Man, van Kooten et al. 1989, Leite, Rodrigues et al. 2017, 511 Higuchi, Rodrigues et al. 2018). LPS of Gram-negative species, a pro512 inflammatory endotoxin, binds to TLR-4 in the gastrointestinal mucosa, triggering 513 an inflammatory cascade that causes localised NF- $\kappa \mathrm{B}$ activation, ensuing the 514 systemic secretion of IL-6 (Steimle, Michaelis et al. 2019).

515 Taken together, we proffer that in the setting of the enfeebled mucosal barrier, the 516 overload of Gram-negative bacteria activates the gut innate immune system, 517 generating IL-6-driven systemic low-grade inflammation, ultimately leading to 518 UE. Indeed, Demir et al. found higher serum IL-6 levels, but not TNF- $\alpha$ levels, in 519 women with UE than fertile women (Demir, Guven et al. 2009). Since elevated IL5206 levels impair various aspects of reproductive physiology, including LH secretion, 521 LH-induced ovulation, and FSH-stimulated E2 and progesterone release, the gut 
522 bacteria-induced higher IL-6 levels may thus cause UE through these mechanisms

523 (Demir, Guven et al. 2009).

524 In the infertile cohort, fascinatingly, elevated levels of Hungatella, producers of 525 trimethylamine $\mathrm{N}$-oxide (TMAO), which enhances thrombotic potential through 526 platelet hyperreactivity, were found than the controls (Zhu, Gregory et al. 2016,

527 Genoni, Christophersen et al. 2019). This data raises the possibility that an 528 overactive coagulation system is a common mechanism of implantation failure. 529 Since levels of Hungatella were highest in the UE group, this indicates an 530 important role of thrombosis in UE. Indeed, Azem et al. found inherited 531 thrombophilia plays a role in repeated IVF failures, particularly in the subgroup 532 with UE (Azem, Maslovich et al. 2001).

533 Regarding the landscape of the vaginal microbiota, data showed that the vaginal 534 bacterial community was less diverse than in the gut. Rarefaction analysis found 535 that the RIF group had the lowest microbial diversity of the three groups, 536 suggesting a healthy vaginal microbiota in the RIF group (Lokken, Richardson et 537 al. 2019). We posit that through TNF- $\alpha$-driven systemic insulin resistance, gut 538 dysbiosis in the RIF group causes hyperglycemia and consequently increases 539 glycogen levels in the vaginal epithelium, which is required for the maintenance of 540 healthy microbiota (Carrara, Bazotte et al. 2009, Amabebe and Anumba 2018). 541 Alternatively, adipose tissue-driven rise in peripheral estrogen may increase the 
542 glycogen content of vaginal epithelial cells (Lokken, Richardson et al. 2019). This

543 chimes with the finding that obesity protects against vaginal dysbiosis (Lokken,

544 Richardson et al. 2019). By contrast, the highest microbial diversity in the control

545 group suggests vaginal dysbiosis. LEfSe analysis found Leptotrichia, an

546 opportunistic pathogen of the female urogenital tract of the phylum Fusobacteria,

547 in this group (Thilesen, Nicolaidis et al. 2007). In line with this, other pathogenic

548 genera such as Gardnerella, Prevotella, and Snaethia were relatively more

549 abundant in the controls compared to the infertile groups (Thilesen, Nicolaidis et

550 al. 2007, Amabebe and Anumba 2018).

551 Consistent with previous research, Firmicutes, mainly Lactobacilli spp.,

552 constituted the bulk of total bacteria of all the groups (Madhivanan, Raphael et al.

553 2014, Madhivanan, Alleyn et al. 2015). Of the nine detected Lactobacilli spp., four

554 belonged to the L. delbrueckii, subsp. of the L. acidophilus group (L. iners,

555 L.gasseri, L. jensenii, L. equicursoris), three belonged to the L. reuteri group ( $L$.

556 fermentum, L. reuteri, L. vaginalis), and two belonged to the L. salivarius group

557 (L.ruminis, L. salivarius) (Salvetti, Torriani et al. 2012). Of the three groups, the $L$.

558 reuteri group comprises mostly obligate heterofermentative lactobacilli, consistent

559 with the finding that compared to the women in the USA, Indian women housed a

560 higher proportion of obligate heterofermentative lactobacilli, reflecting a need for

561 metabolic plasticity and an adaptation against high pathogen load (Salvetti, 
562 Torriani et al. 2012, Madhivanan, Raphael et al. 2014, Madhivanan, Alleyn et al.

563 2015). Three Lactobacillus species dominated the vaginal microbiota across the

564 groups: L. iners, L.gasseri, and L.ruminis, with L. iners being the most abundant, 565 suggesting the existence of community state type 3 (CST 3) of the five human 566 vaginal microbial communities (HVMC) as classified by Ravel et al. (2011) 567 (Ravel, Gajer et al. 2011). To put this in context, L. crispatus, L. jensenii, $L$. 568 gasseri, and $L$. iners occur most frequently in the healthy vagina worldwide, 569 although substantial geographic, socio-economic and ethnic variations exist 570 (Madhivanan, Krupp et al. 2008, Madhivanan, Raphael et al. 2014).

571 By lowering the vaginal $\mathrm{PH}<4$ through lactic acid, generating bacteriocins and 572 hydrogen peroxide $\left(\mathrm{H}_{2} \mathrm{O}_{2}\right)$, or acting as a competitive inhibitor, Lactobacilli spp. 573 protect the vagina from opportunistic pathogens (Kalia, Singh et al. 2020, Wang, 574 Fan et al. 2020). Notably, though this protection varies according to strains based 575 on their ability to produce D-lactic acid, which keeps $\mathrm{PH}<4$, and antibacterial 576 compounds (Wang, Fan et al. 2020). Most studies have shown that L. iners, 577 lacking the ability to produce D-lactic acid, is less protective than other strains 578 such as L. crispatus and L. salivarius (Pino, Bartolo et al. 2019, Kalia, Singh et al. 579 2020, Wang, Fan et al. 2020). Indeed, the highest relative proportions of 580 Lactobacillus iners $A B-1$ occurred in the UE group, suggesting a weakened 581 resistance to colonisation by pathogens (Wang, Fan et al. 2020). 


\subsection{Conclusion}

583 In sum, we illuminated gut and vaginal bacterial communities' landscape, both at

584 broader and finer levels, in implantation failure-associated infertile women and 585 fertile women and offer conjectures to explain the data. We discovered that the 586 infertile cohort had gut dysbiosis. Most importantly, these findings suggest that gut 587 dysbiosis does not necessarily lead to vaginal dysbiosis. We hope that this study 588 has laid the foundation of research on the link between the gut microbiota, the gut589 reproductive microbiota axis, and implantation failure, which can lead microbiota590 based diagnostic tools and therapeutic strategies.

\section{$591 \quad 4.2$ Limitations}

592 Our study has a few limitations. First, since it is an underpowered single-center 593 study, multi-center longitudinal studies with a large sample size are needed.

594 Second, although we suggested the mechanistic hypotheses, we did not measure 595 alterations in the immune system, hormones, platelet parameters and bacterial 596 metabolic products such as short-chain fatty acids. Third, owing to the limited 597 resolution of the $16 \mathrm{~S}$ rRNA-sequencing technique, we could not identify what 598 specific bacterial species or strains were involved. Fourth, we have not controlled 599 for the factors that influence the vaginal microbiota such as menses, vaginal 600 douching, and contraception. Finally, the functional significance of many species 601 such as peptoniphilus remains undetermined in our analysis as the literature is 
602 scant on these genera. Hence, future investigations should address these 603 shortcomings.

604 


\section{References}

Al-Assal, K., A. C. Martinez, R. S. Torrinhas, C. Cardinelli and D. Waitzberg (2018). "Gut microbiota and obesity." Clinical Nutrition Experimental 20: 60-64.

Al-Nasiry, S., E. Ambrosino, M. Schlaepfer, S. A. Morré, L. Wieten, J. W. Voncken, M. Spinelli, M. Mueller and B. W. Kramer (2020). "The Interplay Between Reproductive Tract Microbiota and Immunological System in Human Reproduction." Frontiers in immunology 11: 378-378.

Alexander, K. L., S. R. Targan and C. O. Elson, 3rd (2014). "Microbiota activation and regulation of innate and adaptive immunity." Immunological reviews 260(1): 206-220.

Amabebe, E. and D. O. C. Anumba (2018). "The Vaginal Microenvironment: The Physiologic Role of Lactobacilli." Frontiers in medicine 5: 181-181.

Azad, M. A. K., M. Sarker, T. Li and J. Yin (2018). "Probiotic Species in the Modulation of Gut Microbiota: An Overview." BioMed research international 2018: $9478630-9478630$.

Azem, F., S. Maslovich, A. Amit, J. B. Lessing, M. J. Kupferminc and A. Many (2001). "Increased rates of thrombophilia in women with repeated in-vitro fertilization-embryo transfer (IVF-ET) failures." Fertility and Sterility 76(3): S226.

Baker, J. M., L. Al-Nakkash and M. M. Herbst-Kralovetz (2017). "Estrogen-gut microbiome axis: Physiological and clinical implications." Maturitas 103: 45-53.

Bellver, J., S. R. Soares, C. Alvarez, E. Munoz, A. Ramirez, C. Rubio, V. Serra, J. Remohi and A. Pellicer (2008). "The role of thrombophilia and thyroid autoimmunity in unexplained infertility, implantation failure and recurrent spontaneous abortion." Hum Reprod 23(2): 278284.

Brown, G. C. (2019). "The endotoxin hypothesis of neurodegeneration." Journal of Neuroinflammation 16(1): 180.

Budden, K. F., S. L. Gellatly, D. L. A. Wood, M. A. Cooper, M. Morrison, P. Hugenholtz and P. M. Hansbro (2017). "Emerging pathogenic links between microbiota and the gut-lung axis." Nature Reviews Microbiology 15(1): 55-63.

Carrara, M. A., R. B. Bazotte, L. Donatti, T. I. Svidzinski, M. E. Consolaro, E. V. Patussi and M. R. Batista (2009). "Effect of experimental diabetes on the development and maintenance of vulvovaginal candidiasis in female rats." Am J Obstet Gynecol 200(6): 659 e651-654.

Chang, E. M., J. E. Han, H. H. Seok, D. R. Lee, T. K. Yoon and W. S. Lee (2013). "Insulin resistance does not affect early embryo development but lowers implantation rate in in vitro maturation-in vitro fertilization-embryo transfer cycle." Clinical Endocrinology 79(1): 93-99.

Cho, S. H., J. H. Kim, H. J. An, J. O. Kim, Y. R. Kim, W. S. Lee and N. K. Kim (2019). "Association of methionine synthase (rs1805087), methionine synthase reductase (rs1801394), and methylenetetrahydrofolate dehydrogenase 1 (rs2236225) genetic polymorphisms with recurrent implantation failure." Hum Fertil (Camb): 1-8.

Christiansen, O. B., H. S. Nielsen, A. Kolte and A. T. Pedersen (2006). "Research methodology and epidemiology of relevance in recurrent pregnancy loss." Semin Reprod Med 24(1): 5-16.

Christiansen, O. B., H. S. Nielsen and A. M. Kolte (2006). "Future directions of failed implantation and recurrent miscarriage research." Reprod Biomed Online 13(1): 71-83.

Dai, Z.-L., G. Wu and W.-Y. Zhu (2011). "Amino acid metabolism in intestinal bacteria: links between gut ecology and host health." Frontiers in bioscience (Landmark edition) 16: 17681786. 
de Man, P., C. van Kooten, L. Aarden, I. Engberg, H. Linder and C. Svanborg Edén (1989). "Interleukin-6 induced at mucosal surfaces by gram-negative bacterial infection." Infection and immunity 57(11): 3383-3388.

Demir, B., S. Guven, E. S. G. Guven, Y. Atamer and T. Gul (2009). "Serum IL-6 level may have role in the pathophysiology of unexplained infertility." American journal of reproductive immunology (New York, N.Y. : 1989) 62(4): 261-267.

El Hachem, H., V. Crepaux, P. May-Panloup, P. Descamps, G. Legendre and P.-E. Bouet (2017). "Recurrent pregnancy loss: current perspectives." International journal of women's health 9: 331345.

Genoni, A., C. T. Christophersen, J. Lo, M. Coghlan, M. C. Boyce, A. R. Bird, P. Lyons-Wall and A. Devine (2019). "Long-term Paleolithic diet is associated with lower resistant starch intake, different gut microbiota composition and increased serum TMAO concentrations." European Journal of Nutrition.

Graham, R. A., M. W. Seif, J. D. Aplin, T. C. Li, I. D. Cooke, A. W. Rogers and P. Dockery (1990). "An endometrial factor in unexplained infertility." BMJ (Clinical research ed.) 300(6737): 1428-1431.

Guo, X., J. Li, R. Tang, G. Zhang, H. Zeng, R. J. Wood and Z. Liu (2017). "High Fat Diet Alters Gut Microbiota and the Expression of Paneth Cell-Antimicrobial Peptides Preceding Changes of Circulating Inflammatory Cytokines." Mediators of inflammation 2017: 9474896-9474896.

Hernández-Vargas, P., M. Muñoz and F. Domínguez (2020). "Identifying biomarkers for predicting successful embryo implantation: applying single to multi-OMICs to improve reproductive outcomes." Human Reproduction Update 26(2): 264-301.

Higuchi, B. S., N. Rodrigues, M. I. Gonzaga, J. C. C. Paiolo, N. Stefanutto, W. P. Omori, D. G. Pinheiro, J. L. Brisotti, E. Matheucci, Jr., V. S. Mariano and G. L. V. de Oliveira (2018). "Intestinal Dysbiosis in Autoimmune Diabetes Is Correlated With Poor Glycemic Control and Increased Interleukin-6: A Pilot Study." Frontiers in immunology 9: 1689-1689.

Jang, H. B., M.-K. Choi, J. H. Kang, S. I. Park and H.-J. Lee (2017). "Association of dietary patterns with the fecal microbiota in Korean adolescents." BMC Nutrition 3(1): 20.

Jennings, A., M. Koch, M. K. Jensen, C. Bang, J. Kassubek, H.-P. Müller, U. Nöthlings, A. Franke, W. Lieb and A. Cassidy (2019). "The role of the gut microbiome in the association between habitual anthocyanin intake and visceral abdominal fat in population-level analysis." The American Journal of Clinical Nutrition 111(2): 340-350.

Jinno, M., A. Watanabe, N. Eguchi, N. Hatakeyama, J. Hirohama and E. Hiura (2010). "Lowdose metformin improves pregnancy rate in IVF repeaters without polycystic ovary syndrome: its indication and mechanism." Fertility and Sterility 94(4): S29.

Kaakoush, N. O. (2015). "Insights into the Role of Erysipelotrichaceae in the Human Host." Frontiers in cellular and infection microbiology 5: 84-84.

Kalia, N., J. Singh and M. Kaur (2020). "Microbiota in vaginal health and pathogenesis of recurrent vulvovaginal infections: a critical review." Annals of Clinical Microbiology and Antimicrobials 19(1): 5.

Kamel, R. M. (2010). "Management of the infertile couple: an evidence-based protocol." Reproductive biology and endocrinology : RB\&E 8: 21-21.

Koliada, A., G. Syzenko, V. Moseiko, L. Budovska, K. Puchkov, V. Perederiy, Y. Gavalko, A. Dorofeyev, M. Romanenko, S. Tkach, L. Sineok, O. Lushchak and A. Vaiserman (2017). "Association between body mass index and Firmicutes/Bacteroidetes ratio in an adult Ukrainian population." BMC microbiology 17(1): 120-120. 
Le Chatelier, E., T. Nielsen, J. Qin, E. Prifti, F. Hildebrand, G. Falony, M. Almeida, M. Arumugam, J.-M. Batto, S. Kennedy, P. Leonard, J. Li, K. Burgdorf, N. Grarup, T. Jørgensen, I. Brandslund, H. B. Nielsen, A. S. Juncker, M. Bertalan, F. Levenez, N. Pons, S. Rasmussen, S. Sunagawa, J. Tap, S. Tims, E. G. Zoetendal, S. Brunak, K. Clément, J. Doré, M. Kleerebezem, K. Kristiansen, P. Renault, T. Sicheritz-Ponten, W. M. de Vos, J.-D. Zucker, J. Raes, T. Hansen, H. I. T. c. Meta, P. Bork, J. Wang, S. D. Ehrlich and O. Pedersen (2013). "Richness of human gut microbiome correlates with metabolic markers." Nature 500(7464): 541-546.

Leite, A. Z., N. d. C. Rodrigues, M. I. Gonzaga, J. C. C. Paiolo, C. A. de Souza, N. A. V. Stefanutto, W. P. Omori, D. G. Pinheiro, J. L. Brisotti, E. Matheucci Junior, V. S. Mariano and G. L. V. de Oliveira (2017). "Detection of Increased Plasma Interleukin-6 Levels and Prevalence of Prevotella copri and Bacteroides vulgatus in the Feces of Type 2 Diabetes Patients." Frontiers in immunology 8: 1107-1107.

Lindsay, T. J. and K. R. Vitrikas (2015). "Evaluation and treatment of infertility." American family physician 91(5): 308-314.

Lokken, E. M., B. A. Richardson, J. Kinuthia, K. Mwinyikai, A. Abdalla, W. Jaoko, K. Mandaliya, J. Shafi and R. Scott McClelland (2019). "A Prospective Cohort Study of the Association Between Body Mass Index and Incident Bacterial Vaginosis." Sexually transmitted diseases 46(1): 31-36.

Lopetuso, L. R., F. Scaldaferri, V. Petito and A. Gasbarrini (2013). "Commensal Clostridia: leading players in the maintenance of gut homeostasis." Gut pathogens 5(1): 23-23.

Madhivanan, P., H. N. Alleyn, E. Raphael, K. Krupp, K. Ravi, R. Nebhrajani, A. Arun, A. L. Reingold, L. W. Riley and J. D. Klausner (2015). "Identification of culturable vaginal Lactobacillus species among reproductive age women in Mysore, India." J Med Microbiol 64(6): 636-641.

Madhivanan, P., K. Krupp, V. Chandrasekaran, C. Karat, A. Arun, C. R. Cohen, A. L. Reingold and J. D. Klausner (2008). "Prevalence and correlates of bacterial vaginosis among young women of reproductive age in Mysore, India." Indian J Med Microbiol 26(2): 132-137.

Madhivanan, P., E. Raphael, A. Rumphs, K. Krupp, K. Ravi, V. Srinivas, A. Arun, A. L. Reingold, J. D. Klausner and L. W. Riley (2014). "Characterization of culturable vaginal Lactobacillus species among women with and without bacterial vaginosis from the United States and India: a cross-sectional study." Journal of medical microbiology 63(Pt 7): 931-935.

Mahajan, K. and A. Batra (2018). "Obesity in adult asian indians- the ideal BMI cut-off." Indian heart journal 70(1): 195-195.

Matijašić, B. B., T. Obermajer, L. Lipoglavšek, I. Grabnar, G. Avguštin and I. Rogelj (2014). "Association of dietary type with fecal microbiota in vegetarians and omnivores in Slovenia." European Journal of Nutrition 53(4): 1051-1064.

Mejía-León, M. E. and A. M. C. d. 1. Barca (2015). "Diet, Microbiota and Immune System in Type 1 Diabetes Development and Evolution." Nutrients 7(11): 9171-9184.

Moreno, I., F. M. Codoñer, F. Vilella, D. Valbuena, J. F. Martinez-Blanch, J. Jimenez-Almazán, R. Alonso, P. Alamá, J. Remohí, A. Pellicer, D. Ramon and C. Simon (2016). "Evidence that the endometrial microbiota has an effect on implantation success or failure." Am J Obstet Gynecol 215(6): 684-703.

Okumura, R. and K. Takeda (2017). "Roles of intestinal epithelial cells in the maintenance of gut homeostasis." Experimental \& Molecular Medicine 49(5): e338-e338.

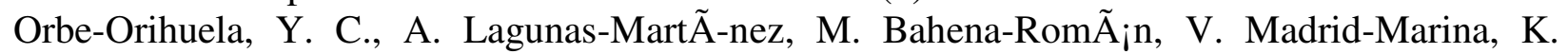
Torres-Poveda, E. Flores-Alfaro, A. MÃđ@ndez-PadrÃn, C. E. DÃ-az-BenÃ-tez, O. Peralta- 
Zaragoza, D. Ant $\tilde{A}^{\circ}$ nez-Ortiz, M. Cruz and A. I. Burguete-GarcÃ-a (2018). "High relative abundance of firmicutes and increased TNF- \pm levels correlate with obesity in children." Salud Publica Mex. 2018; 60(1): 5-11.

Ott, S. J. and S. Schreiber (2006)."Reduced microbial diversity in inflammatory bowel diseases." Gut 55(8): 1207-1207.

Pino, A., E. Bartolo, C. Caggia, A. Cianci and C. L. Randazzo (2019). "Detection of vaginal lactobacilli as probiotic candidates." Scientific Reports 9(1): 3355.

Polanski, L. T., M. N. Baumgarten, S. Quenby, J. Brosens, B. K. Campbell and N. J. RaineFenning (2014). "What exactly do we mean by 'recurrent implantation failure'? A systematic review and opinion." Reproductive BioMedicine Online 28(4): 409-423.

Ravel, J. and R. M. Brotman (2016). "Translating the vaginal microbiome: gaps and challenges." Genome medicine 8(1): 35-35.

Ravel, J., P. Gajer, Z. Abdo, G. M. Schneider, S. S. K. Koenig, S. L. McCulle, S. Karlebach, R. Gorle, J. Russell, C. O. Tacket, R. M. Brotman, C. C. Davis, K. Ault, L. Peralta and L. J. Forney (2011). "Vaginal microbiome of reproductive-age women." Proceedings of the National Academy of Sciences of the United States of America 108 Suppl 1(Suppl 1): 4680-4687.

Salvetti, E., S. Torriani and G. E. Felis (2012). "The Genus Lactobacillus: A Taxonomic Update." Probiotics Antimicrob Proteins 4(4): 217-226.

Sanz, Y., A. Santacruz and G. De Palma (2008). "Insights into the roles of gut microbes in obesity." Interdisciplinary perspectives on infectious diseases 2008: 829101-829101.

Sekirov, I., S. L. Russell, L. C. M. Antunes and B. B. Finlay (2010). "Gut Microbiota in Health and Disease." Physiological Reviews 90(3): 859-904.

Simon, A. and N. Laufer (2012). "Assessment and treatment of repeated implantation failure (RIF)." Journal of assisted reproduction and genetics 29(11): 1227-1239.

Steimle, A., L. Michaelis, F. Di Lorenzo, T. Kliem, T. Münzner, J. K. Maerz, A. Schäfer, A. Lange, R. Parusel, K. Gronbach, K. Fuchs, A. Silipo, H. H. Öz, B. J. Pichler, I. B. Autenrieth, A. Molinaro and J.-S. Frick (2019). "Weak Agonistic LPS Restores Intestinal Immune Homeostasis." Molecular therapy : the journal of the American Society of Gene Therapy 27(11): 1974-1991.

Tersigni, C., S. D'Ippolito, F. Di Nicuolo, R. Marana, V. Valenza, V. Masciullo, F. Scaldaferri, F. Malatacca, C. de Waure, A. Gasbarrini, G. Scambia and N. Di Simone (2018). "Recurrent pregnancy loss is associated to leaky gut: a novel pathogenic model of endometrium inflammation?" Journal of translational medicine 16(1): 102-102.

Thilesen, C. M., M. Nicolaidis, J. E. Lökebö, E. Falsen, A. T. Jorde and F. Müller (2007). "Leptotrichia amnionii, an emerging pathogen of the female urogenital tract." Journal of clinical microbiology 45(7): 2344-2347.

Uchida, M., E. Oyanagi, N. Kawanishi, M. Iemitsu, M. Miyachi, M. J. Kremenik, S. Onodera and H. Yano (2014). "Exhaustive exercise increases the TNF- $\alpha$ production in response to flagellin via the upregulation of toll-like receptor 5 in the large intestine in mice." Immunology letters 158(1-2): 151-158.

Verdam, F. J., S. Fuentes, C. de Jonge, E. G. Zoetendal, R. Erbil, J. W. Greve, W. A. Buurman, W. M. de Vos and S. S. Rensen (2013). "Human intestinal microbiota composition is associated with local and systemic inflammation in obesity." Obesity 21(12): E607-E615.

Vesth, T., A. Ozen, S. C. Andersen, R. S. Kaas, O. Lukjancenko, J. Bohlin, I. Nookaew, T. M. Wassenaar and D. W. Ussery (2013). "Veillonella, Firmicutes: Microbes disguised as Gram negatives." Standards in genomic sciences 9(2): 431-448. 
Vinchi, F. (2019). "Thrombosis Prevention: Let's Drug the Microbiome!" HemaSphere 3(1): e165-e165.

789 Vital, M., A. Karch and D. H. Pieper (2017). "Colonic Butyrate-Producing Communities in 790 Humans: an Overview Using Omics Data." mSystems 2(6): e00130-00117.

791 Wang, C., A. Fan, H. Li, Y. Yan, W. Qi, Y. Wang, C. Han and F. Xue (2020). "Vaginal bacterial 792 profiles of aerobic vaginitis: a case-control study." Diagnostic Microbiology and Infectious 793 Disease 96(4): 114981.

794 Wang, M., S. Wichienchot, X. He, X. Fu, Q. Huang and B. Zhang (2019). "In vitro colonic 795 fermentation of dietary fibers: Fermentation rate, short-chain fatty acid production and changes 796 in microbiota." Trends in Food Science \& Technology 88: 1-9.

797 Wang, Z., S. Saha, S. Van Horn, E. Thomas, C. Traini, G. Sathe, D. K. Rajpal and J. R. Brown 798 (2018). "Gut microbiome differences between metformin- and liraglutide-treated T2DM 799 subjects." Endocrinol Diabetes Metab 1(1): e00009.

800 Winger, E. E., J. L. Reed, S. Ashoush, T. El-Toukhy, S. Ahuja and M. Taranissi (2011). "Degree 801 of TNF- $\alpha /$ IL-10 Cytokine Elevation Correlates With IVF Success Rates in Women Undergoing 802 Treatment With Adalimumab (Humira) and IVIG." American Journal of Reproductive 803 Immunology 65(6): 610-618.

804 Witkin, S. S., K. M. Sultan, G. S. Neal, J. Jeremias, J. A. Grifo and Z. Rosenwaks (1994). 805 "Unsuspected Chlamydia trachomatis infection and in vitro fertilization outcome." American 806 Journal of Obstetrics and Gynecology 171(5): 1208-1214.

807 Zhang, J., H. Liu, X. Mao, Q. Chen, Y. Fan, Y. Xiao, Y. Wang and Y. Kuang (2019). "Effect of 808 body mass index on pregnancy outcomes in a freeze-all policy: an analysis of 22,043 first 809 autologous frozen-thawed embryo transfer cycles in China." BMC Medicine 17(1): 114.

Zhu, W., J. C. Gregory, E. Org, J. A. Buffa, N. Gupta, Z. Wang, L. Li, X. Fu, Y. Wu, M. Mehrabian, R. B. Sartor, T. M. McIntyre, R. L. Silverstein, W. H. W. Tang, J. A. DiDonato, J. M. Brown, A. J. Lusis and S. L. Hazen (2016). "Gut Microbial Metabolite TMAO Enhances Platelet Hyperreactivity and Thrombosis Risk." Cell 165(1): 111-124. 
815 We presented a portion of the data of this study in 816 the 4th World Congress on Recurrent Pregnancy Loss (WCRPL 2019) in 817 Jerusalem, Israel.

818

819 Acknowledgements:

820 The authors wish to acknowledge the technical support of Nitin Savaliya, of 821 Gujarat Biotechnology Research Centre (GBRC), for fecal and vaginal samples 822 sequencing run. We are also grateful to Dr Yuvraj Jadeja, Dr Nipa Shah, Dr Nikita 823 Daswani, Hansa Khrishti and Harmi Thakkar for supporting the clinical work.

824 Availability of data and materials:

825 The 16S rRNA gene sequencing data for all the gut and vaginal microbiota 826 samples analyzed in this study have been deposited with the National Center for 827 Biotechnology Information (NCBI): reference number PRJNA7020230. 


\section{Authors' contributions:}

831

832 BP conceptualized the idea, contributed to the design, made a few suggestions on 833 the statistical methods, interpreted the data, and wrote the manuscript except for 834 the metagenomics part of the method section. NP contributed to the design, patient 835 identification, sample collection, ethical approval, financially supported the clinical 836 work, presented a part of the work, and critically discussed and corrected the 837 manuscript's clinical aspects. NP collected fecal and vaginal samples, performed 838 DNA isolation, sequencing, assisted in the analysis, drafted a part of the material 839 and method section, and prepared some graphs and tables. SP collected fecal and 840 vaginal samples, performed DNA isolation, library preparation of samples, and 841 contributed to the correction of materials and method section. NN analysed the 842 metagenomics data and contributed to the writing of the metagenomics section of 843 the manuscript. RP sequenced fecal samples and corrected material and method 844 section. CJ, the director of GBRC, provided financial and technical support and 845 guidance for metagenomics sequencing and critically discussed the data. NP and 846 MP contributed to ethical permission, patient selection and clinical data acquisition 847 and critically discussed the data. All the authors provided criticisms and read and 848 approved the manuscript. 


\section{Funding:}

851 No external source of funding. The clinical aspect of this work was funded by Dr.

852 Nayna Patel, the director of Akanksha Hospital and Research Institute. The 853 metagenomics aspect of the study was funded by Dr. Chaitanya Joshi, the director 854 of Gujarat Biotechnology Research Centre (GBRC). We report no conflict of 855 interest.

856

857 Competing interests:

858 The authors declare no competing interests.

859

860 Consent for publication:

861 All authors have given their consent for the publication of this manuscript. 
864 Table 1. Study characteristics of the control, RIF, and the UE groups. Data are 865 expressed as the mean $\pm \mathrm{SD}$ or $\mathrm{n} / \mathrm{N}(\%) . \mathrm{RIF}$, recurrent implantation failure; UE, 866 unexplained infertility; BMI, body-mass index; Veg, Vegetarian; NA, Not 867 applicable. Differences between the 3 groups were assessed by 1-factor ANOVA 868 and Tukey's multiple comparison test was used for post-hoc comparisons, if $869 \mathrm{P}<0.05$. In the RIF group, two participants belonged to the RPL category. a. 870 statistically significant difference between CON and RIF.

872 Figure 1. Box plots of $\alpha$-diversity indices of the gut bacterial microbiomes of the

873 control (CON, $\mathrm{N}=11)$, RIF (RIF, $\mathrm{N}=10$ ), and the UE (UE, $\mathrm{N}=10$ ) groups: (a)

874 Shannon and (b) Chao 1 indices. The $P$ values were determined by the Kruskal875 Wallis test.

876

877 Figure 2. Differences in community composition ( $\beta$-diversity) between the control 878 (CON, Red, $\mathrm{N}=11$ ), RIF (RIF, Green, $\mathrm{N}=10$ ), and the UE group (UE, Cyan $879 \mathrm{~N}=10)$ groups. Comparisons are based on the PCoA plots of Bray-Curtis (left) and 880 Jaccard distances (right). Each principal coordinate axis represents the proportion 881 of variance. Non-parametric multivariate analysis of variance (PERMANOVA) 
882 was used to calculate statistical differences between the groups. In the Bray-Curtis-

883 based PCoA, the first and second axes of the PCoA accounted for $21.5 \%$ of the

884 total variance (PERMANOVA, $\mathrm{P}<0.05, \mathrm{R} 2=0.12$ ). In the Jaccard based PCoA, the 885 first and second axes explained $23.4 \%$ of the total variance (PERMANOVA, $886 \mathrm{P}<0.05, \mathrm{R} 2=0.10)$.

887

888 Figure 3. The bar chart shows the comparisons of relative abundances of top gut 889 bacterial taxa between control ( $\mathrm{CON}, \mathrm{N}=11)$, RIF (RIF, $\mathrm{N}=10)$, and the UE (UE, $890 \mathrm{~N}=10$ ) groups at (a) the phylum (b) family and (c) genus levels. No differences in 891 relative abundance were found between the phylum and family levels $(\mathrm{P}>0.05$, 892 Kruskal-Wallis test). Six bacterial genera showed statistically significantly 893 ( $\mathrm{P}<0.05$, Kruskal-wallis test $)$ differential abundance across the three groups are 894 shown.

895

896 Figure 4. Distinct taxa of the gut bacteria determined by linear discriminant 897 analysis effect size (LEfSe) analysis in the control (CON, $N=11), \mathrm{RIF}(\mathrm{RIF}, \mathrm{N}=$ 898 10), and the UE (UE, $\mathrm{N}=10$ ) groups. (a) The cladogram shows the taxa that were 899 significantly elevated between the groups (b) Taxa with an LDA score significant 900 threshold $>3$ are shown $(\mathrm{P}<.05$; LDA score 3$)$. 
902 Figure 5. The bar charts show taxonomic comparisons of the vaginal bacteria 903 between the control (CON, $\mathrm{N}=8$ ), RIF (RIF, $\mathrm{N}=8$ ), and the UE (UE, $\mathrm{N}=8$ )

904 groups at (a) the phylum, (b) family (c) genus level (d) species levels. No 905 differences in relative abundance were found at any levels $(P>0.05$, Kruskal906 Wallis test).

907

908 Figure 6. Differentially abundant vagina bacteria between the control $(\mathrm{CON}, \mathrm{N}=$ 909 8), RIF (RIF, $\mathrm{N}=8$ ), and the UE (UE, $\mathrm{N}=8$ ) groups as determined by linear 910 discriminant analysis effect size (LEfSe) analysis. Taxa with a LDA score 911 significant threshold $>3$ are shown $(\mathrm{P}<.05$; LDA score 3$)$.

912

913 Figure 7. The bar charts show taxonomic comparisons of different Lactobacillus 914 spp. of the vagina between the control ( $\mathrm{CON}, \mathrm{N}=8), \mathrm{RIF}(\mathrm{RIF}, \mathrm{N}=8)$, and the UE 915 (UE, N=8) groups. No significant differences in relative abundance were found 916 between at any levels $(P>0.05$, Kruskal-Wallis test $)$. 917 


\section{Supplementary figures}

920 Figure 1S. Rarefaction analysis of Gut and Vaginal Microbial diversity of the 921 controls, RIF and UE groups. (A) Rarefaction curve of $\alpha$-diversity of the gut 922 bacteria in control ( $\mathrm{CON}, \mathrm{N}=11)$, RIF (RIF, $\mathrm{N}=10$ ), and the UE (UE, $\mathrm{N}=10$ )

923 groups (B) Rarefaction curve of $\alpha$-diversity of the vaginal bacteria in control 924 (CON, $\mathrm{N}=8$ ), RIF (RIF, $\mathrm{N}=8$ ), and the UE (UE, $\mathrm{N}=8$ ) groups. The $\mathrm{x}$-axis shows 925 the number of sequences per sample, and the y-axis shows the rarefaction measure. 926 When the curve plateaus, it shows that the sequencing data volume is sufficient to 927 reveal most of the microbial information in the sample. The values of the y-axis 928 reflect the community diversity of microbiota. The sequence number in the chart 929 shows the sequence number of the sample. OTU, operational taxonomic unit.

930

931 Figure 2S. Rarefaction analysis of microbiome diversity sequences per sample: (a) 932 gut $(\mathrm{n}=31)$ and $(\mathrm{b})$ vaginal samples $(\mathrm{n}=24)$ (Operational taxonomic units (OTUs) 933 for each sample at $97 \%$ of similarity). Each graph represents mean (column) and 934 SD (bars).

Figure 3S. Influence of diet on $\alpha$-diversity between vegetarian $(n=21)$ and non937 vegetarian participants $(n=10)$. No significant difference in $\alpha$-diversity between 938 these groups was found $(P>0.05$, the Mann-Whitney $\mathrm{U}$ test $)$. 
939 Figure 4S. The bar chart shows taxonomic comparisons of the gut bacteria 940 between the controls ( $\mathrm{CON}, \mathrm{N}=11)$ and the infertile cohort (the RIF plus UE 941 groups, $\mathrm{N}=20)$ at the genus level with statistical significance values $(P>0.05$, 942 Mann-Whitney U test).

943

944

945

946

947

948

949

950

951

952

953

954

955

956

957 
Table 1

\begin{tabular}{|c|c|c|c|c|c|c|}
\hline Characteristics & \multicolumn{2}{|c|}{$\begin{array}{r}\text { Control } \\
(\mathrm{n}=11)\end{array}$} & \multicolumn{2}{|c|}{$\begin{array}{l}\text { RIF } \\
(\mathrm{n}=10)\end{array}$} & \multicolumn{2}{|c|}{$\begin{array}{c}\mathrm{UE} \\
(\mathrm{n}=10)\end{array}$} \\
\hline $\begin{array}{c}\text { Age } \\
\text { (Years) }\end{array}$ & \multicolumn{2}{|c|}{$27.9 \pm 3.8$} & \multicolumn{2}{|c|}{$34.5 \pm 4.8^{\mathrm{a}}$} & \multicolumn{2}{|c|}{$30.8 \pm 3.42$} \\
\hline $\begin{array}{c}\text { BMI } \\
\left(\mathrm{kg} / \mathrm{m}^{2}\right)\end{array}$ & \multicolumn{2}{|c|}{$21.62 \pm 4.05$} & \multicolumn{2}{|c|}{$25.9 \pm 3.31^{\mathrm{a}}$} & \multicolumn{2}{|c|}{$23.92 \pm 3$} \\
\hline Nulligravida & \multirow{2}{*}{\multicolumn{2}{|c|}{ NA }} & \multicolumn{2}{|c|}{$2(20 \%)$} & \multicolumn{2}{|c|}{ NA } \\
\hline Nullipara & & & \multicolumn{2}{|c|}{$8(80 \%)$} & \multicolumn{2}{|c|}{$10(100 \%)$} \\
\hline \multirow{2}{*}{ Dietary Information } & Veg & Non-Veg & Veg & Non-Veg & Veg & Non-Veg \\
\hline & $\begin{array}{c}5 \\
(45 \%)\end{array}$ & $\begin{array}{c}6 \\
(54 \%)\end{array}$ & $\begin{array}{c}4 \\
(40 \%)\end{array}$ & $\begin{array}{c}6 \\
(60 \%)\end{array}$ & $\begin{array}{c}9 \\
(90 \%)\end{array}$ & $\begin{array}{c}1 \\
(10 \%)\end{array}$ \\
\hline
\end{tabular}


Figure 1(a)

Kruskal-Wallis , $\mathrm{P}=0.049 ; \mathrm{H}=6.00$

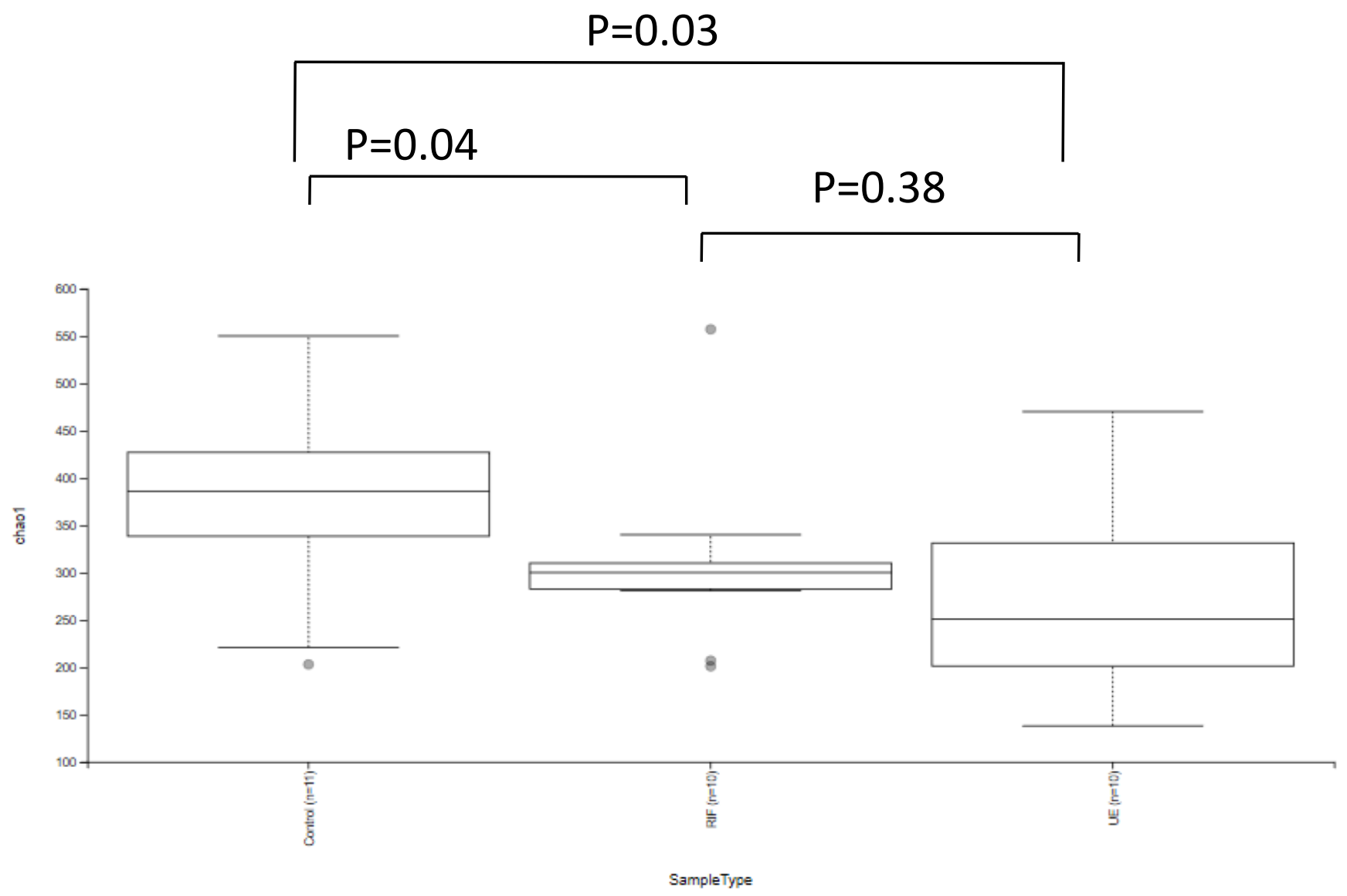


Figure 1(b)

Kruskal-Wallis , $\mathrm{P}=0.003 ; \mathrm{H}=11.6$

$\mathrm{P}=0.002$
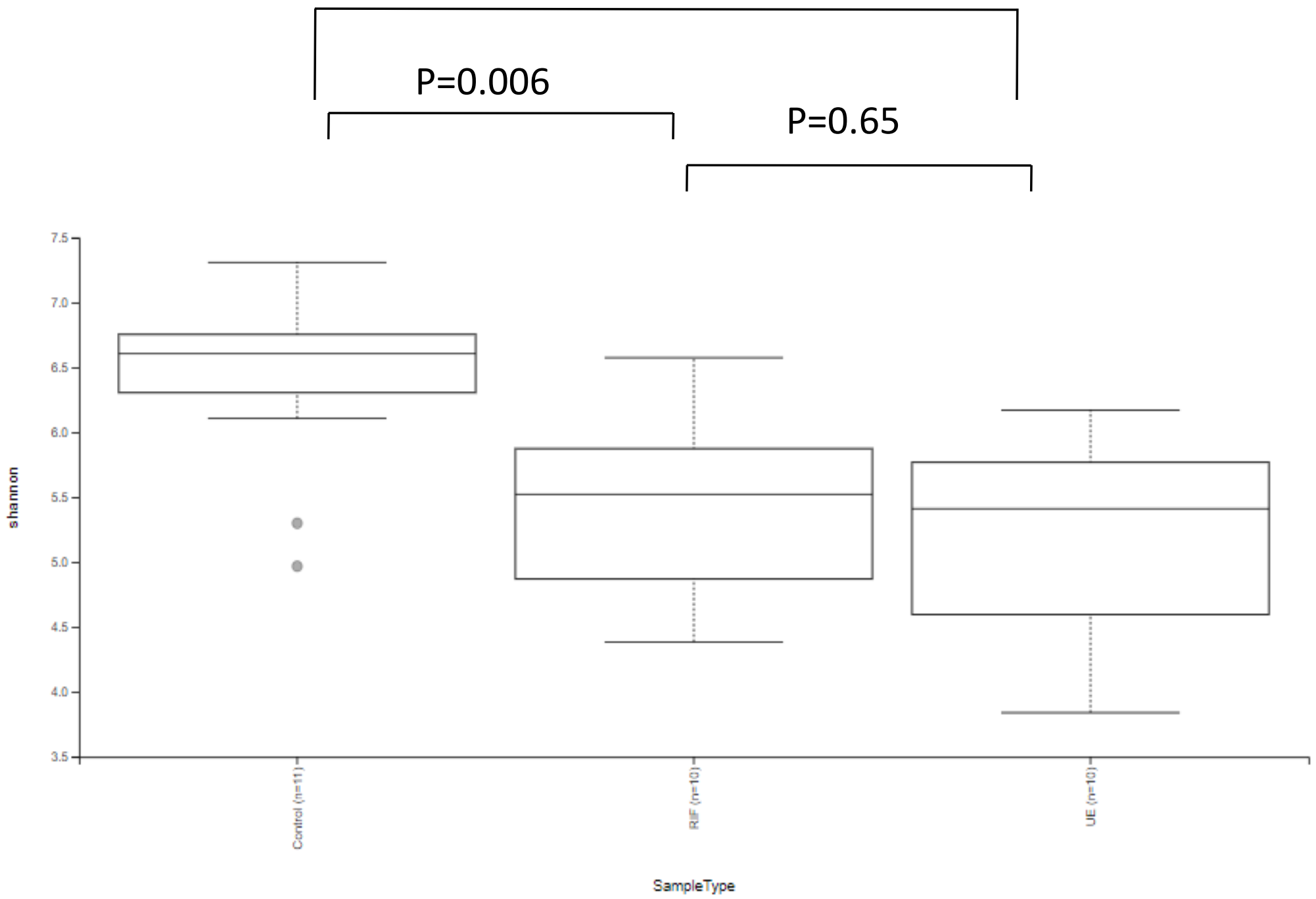


\section{Figure 2(a),(b)}
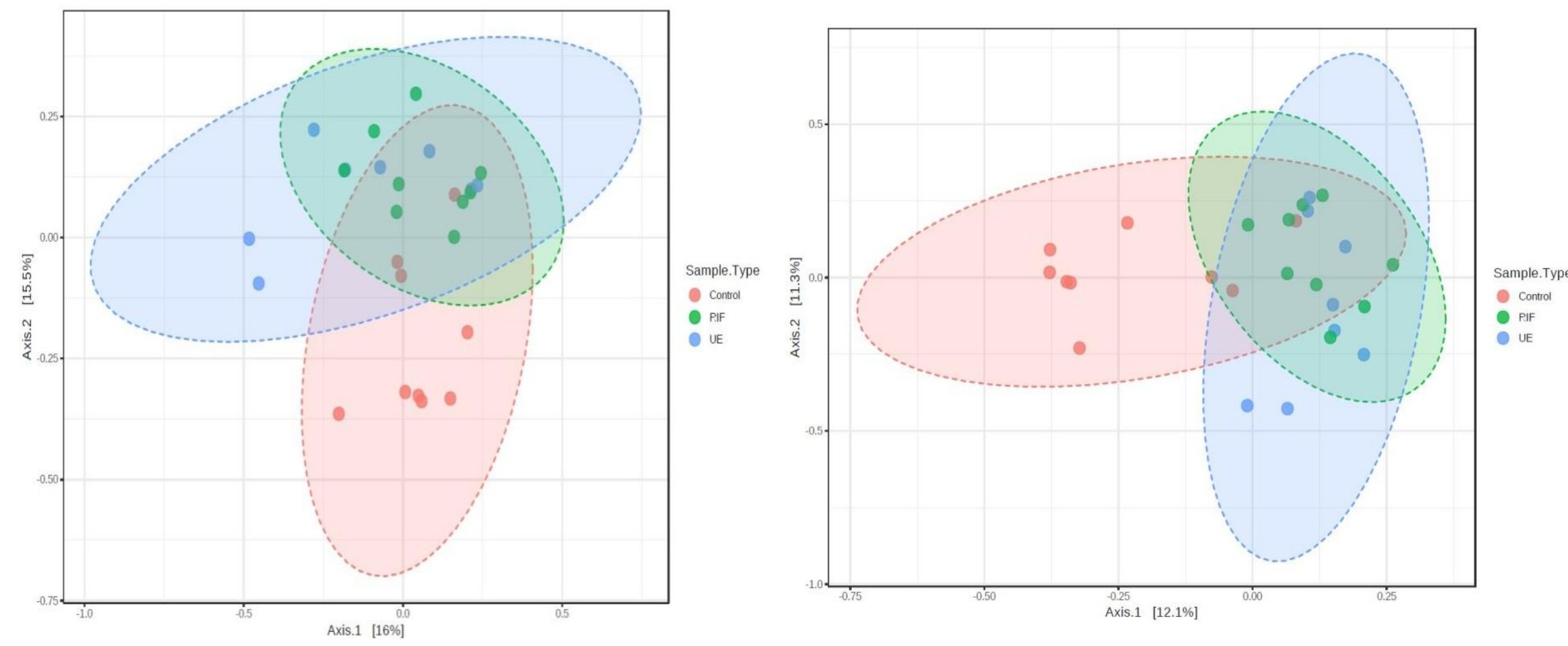
Figure 3(a)

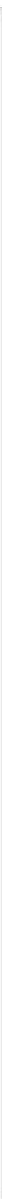


Figure $3(b)$

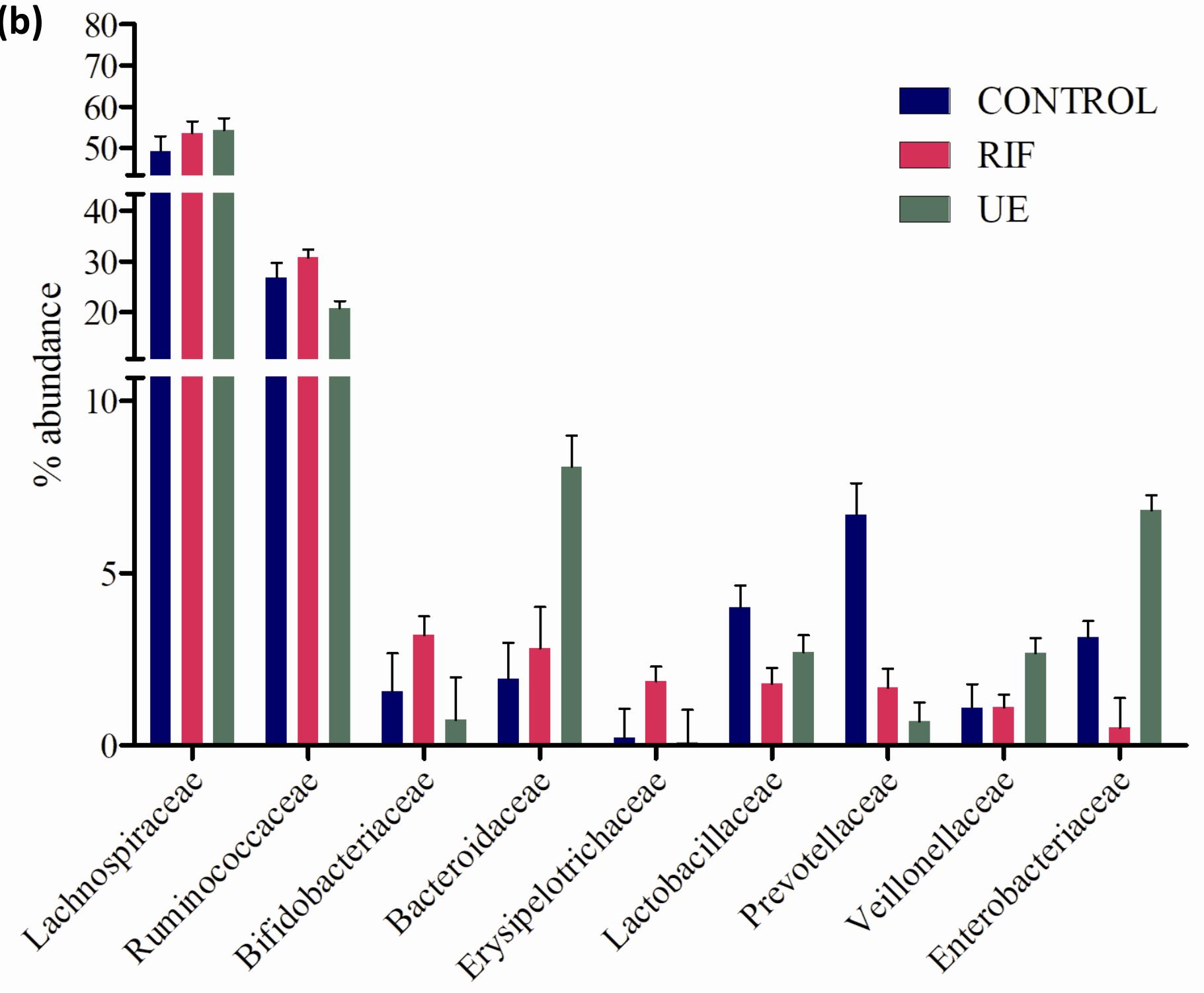


Figure 3(c)

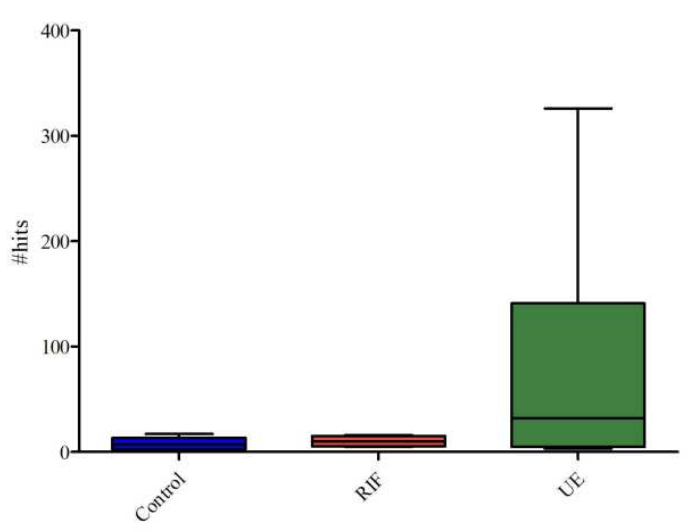

Hungatella

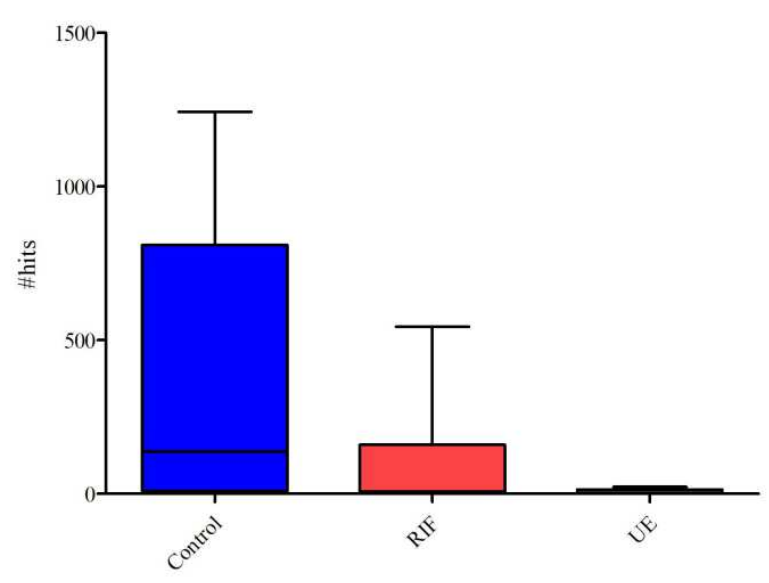

Prevotella_9

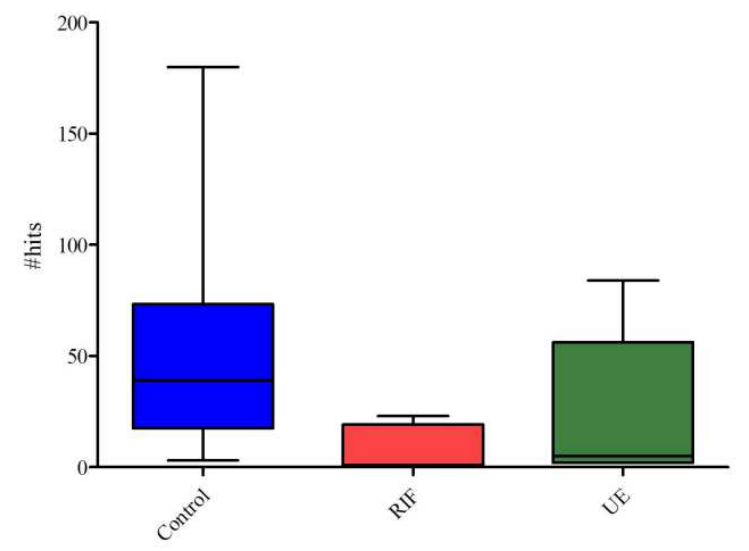

Ruminococcaceae UCG-010

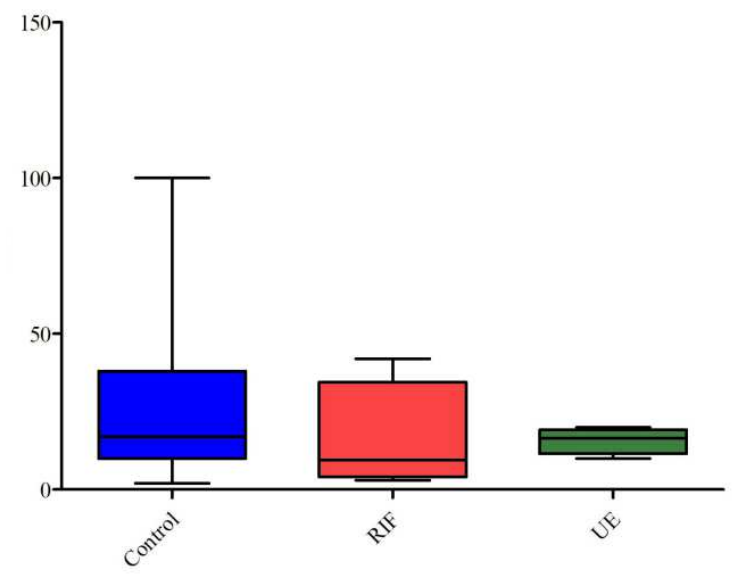

Ruminococcaceae UCG-004

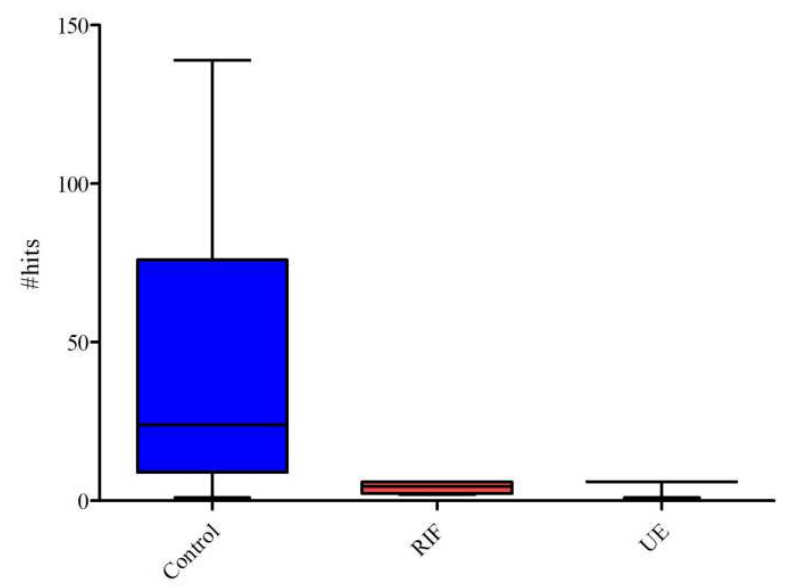

Sutterella 
Figure 4(a)
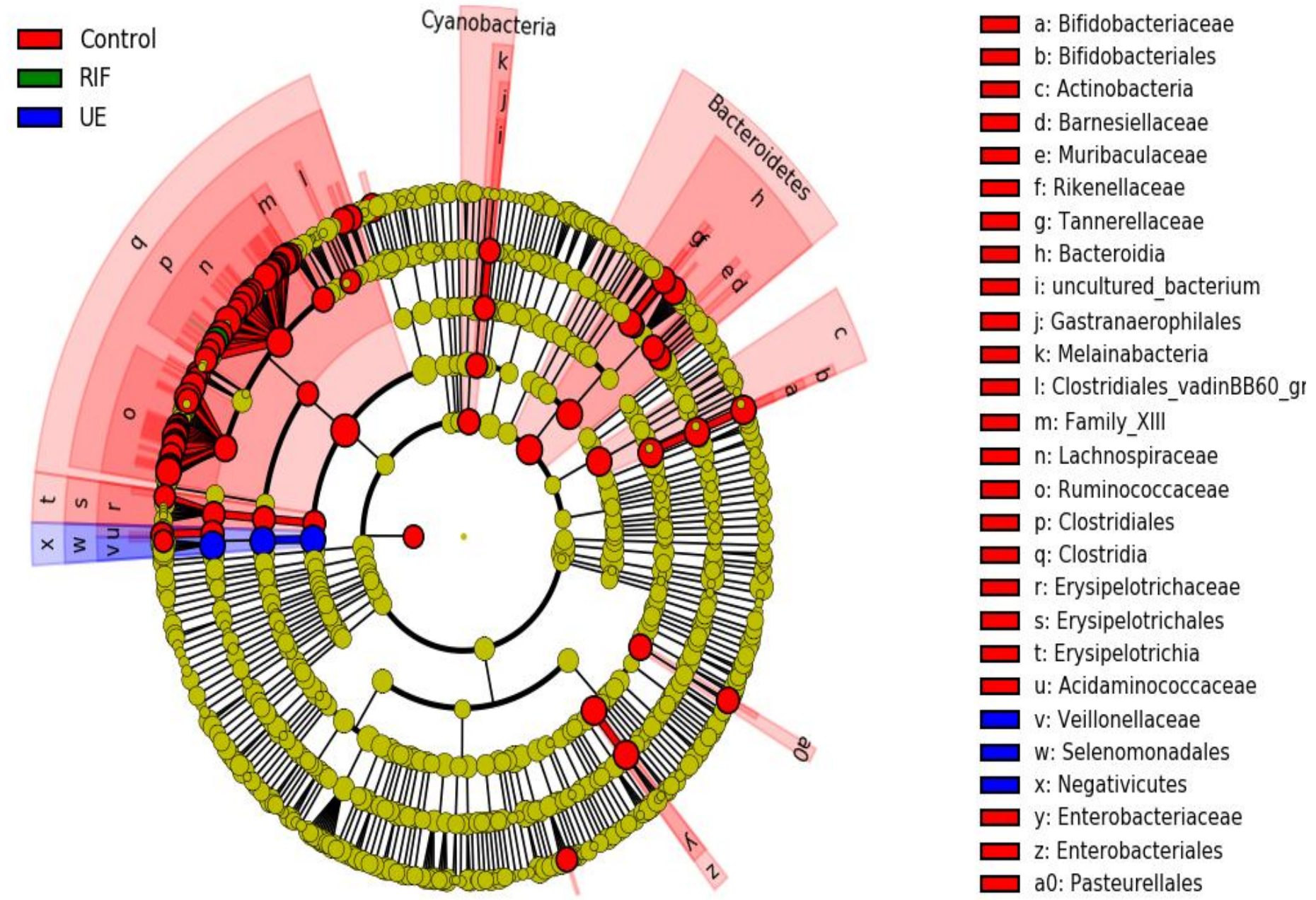
Figure 4(b)
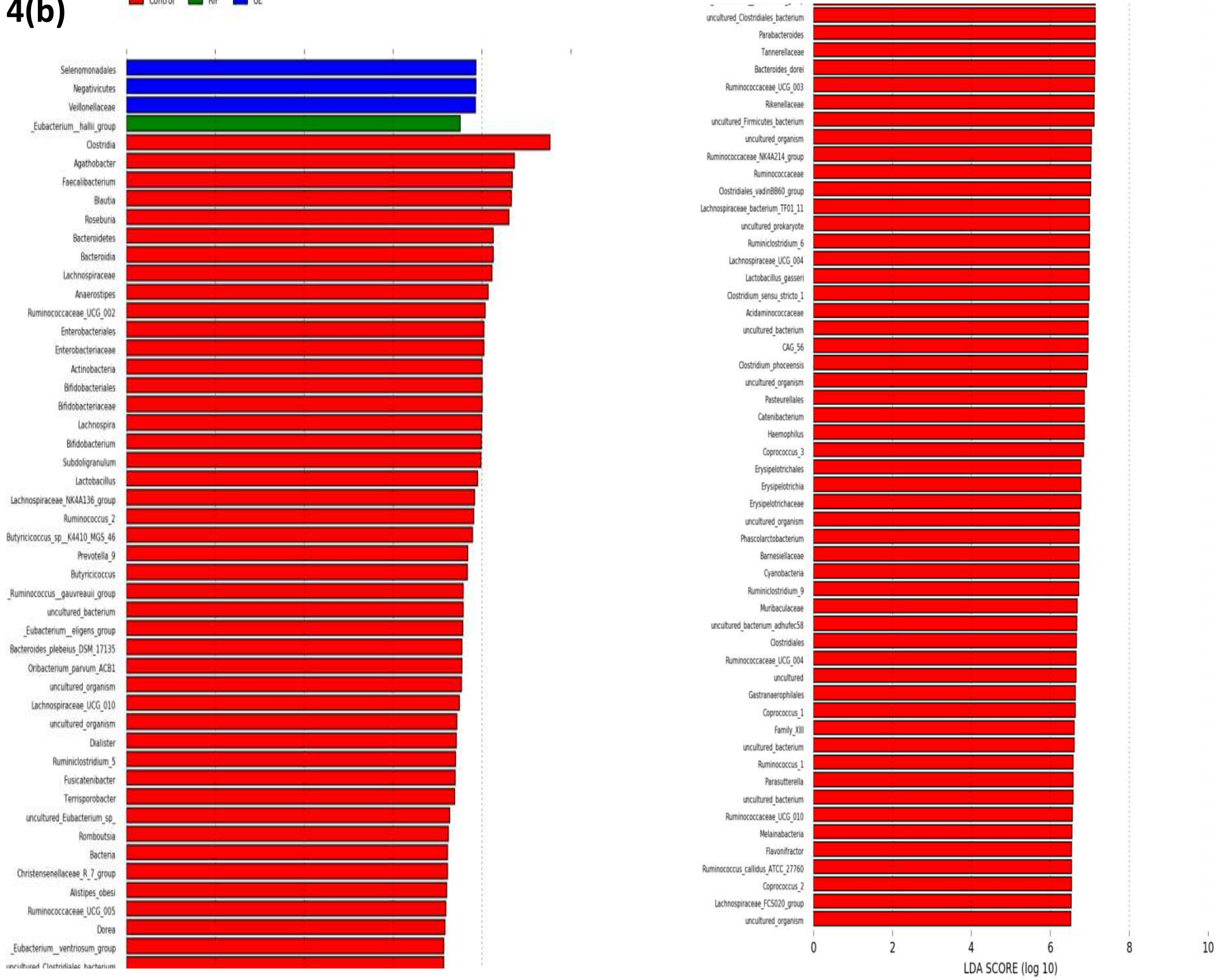
Figure 5(a)

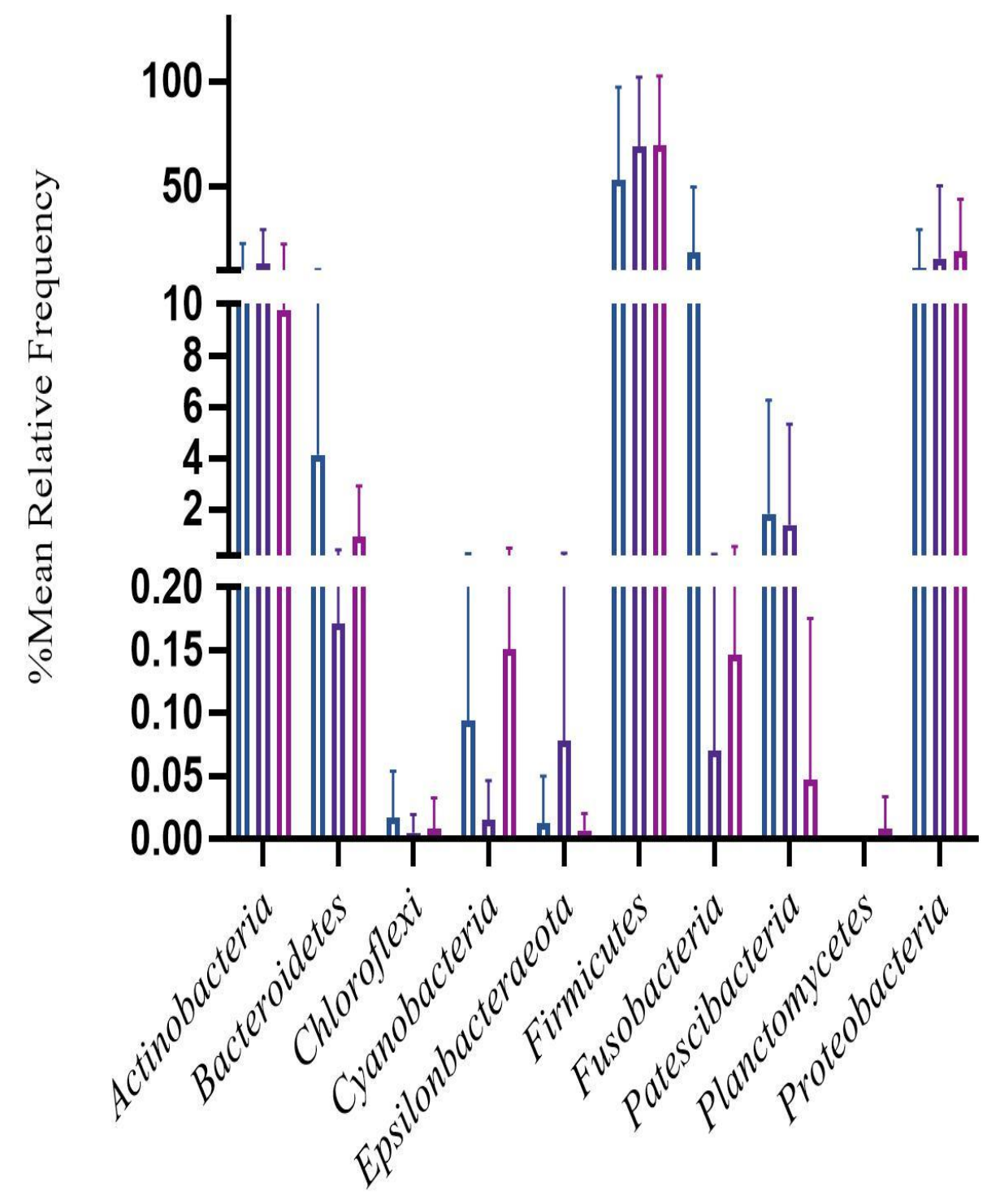

$\square$ CONTROL: mean rel. freq. (\%)

$\square$ RIF: mean rel. freq. (\%)

$\square$ UE: mean rel. freq. (\%) 
Figure 5(b)

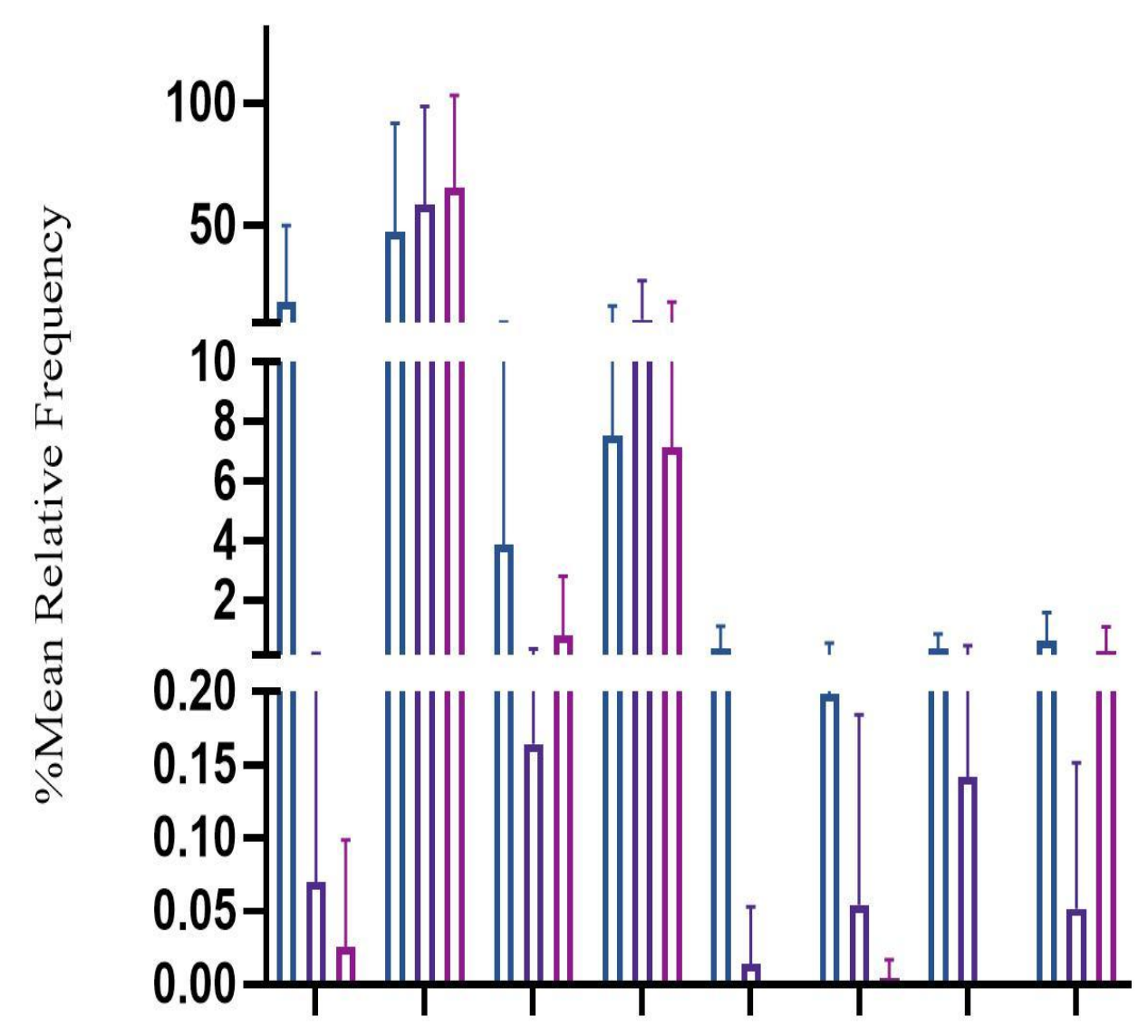

$\square$ CONTROL: mean rel. freq. (\%)

$\square$ RIF: mean rel. freq. (\%)

$\square$ UE: mean rel. freq. (\%)

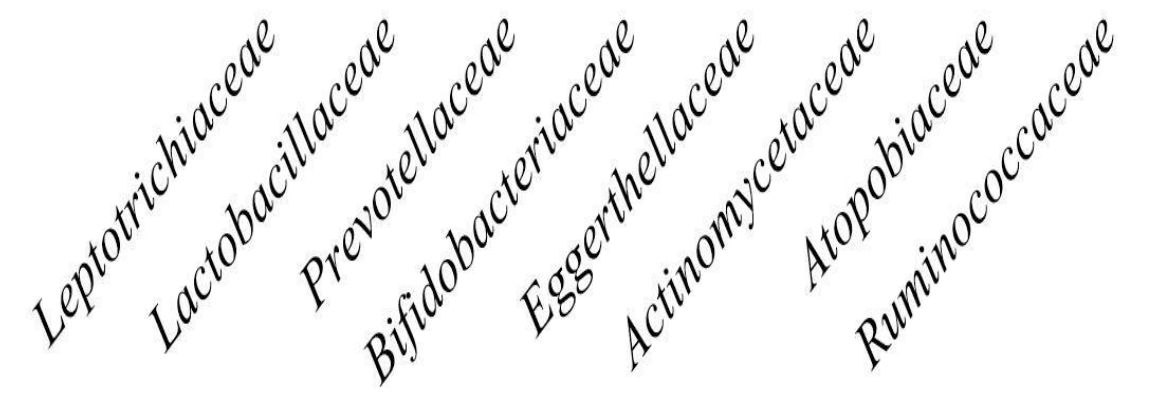




\section{Figure 5(c)}

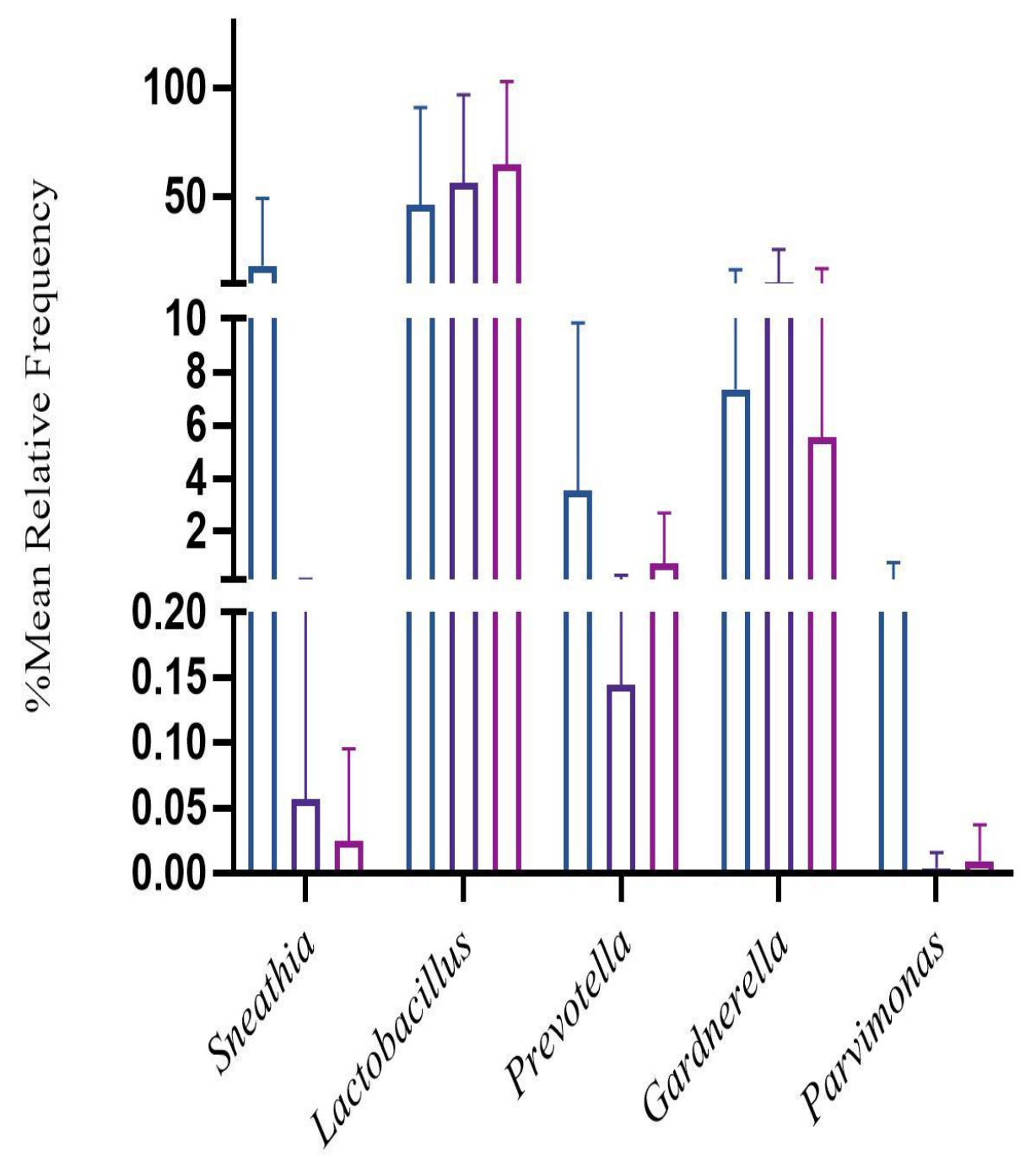

$\square$ CONTROL: mean rel. freq. (\%)

$\square$ RIF: mean rel. freq. (\%)

$\square$ UE: mean rel. freq. (\%) 
Figure 5(d)

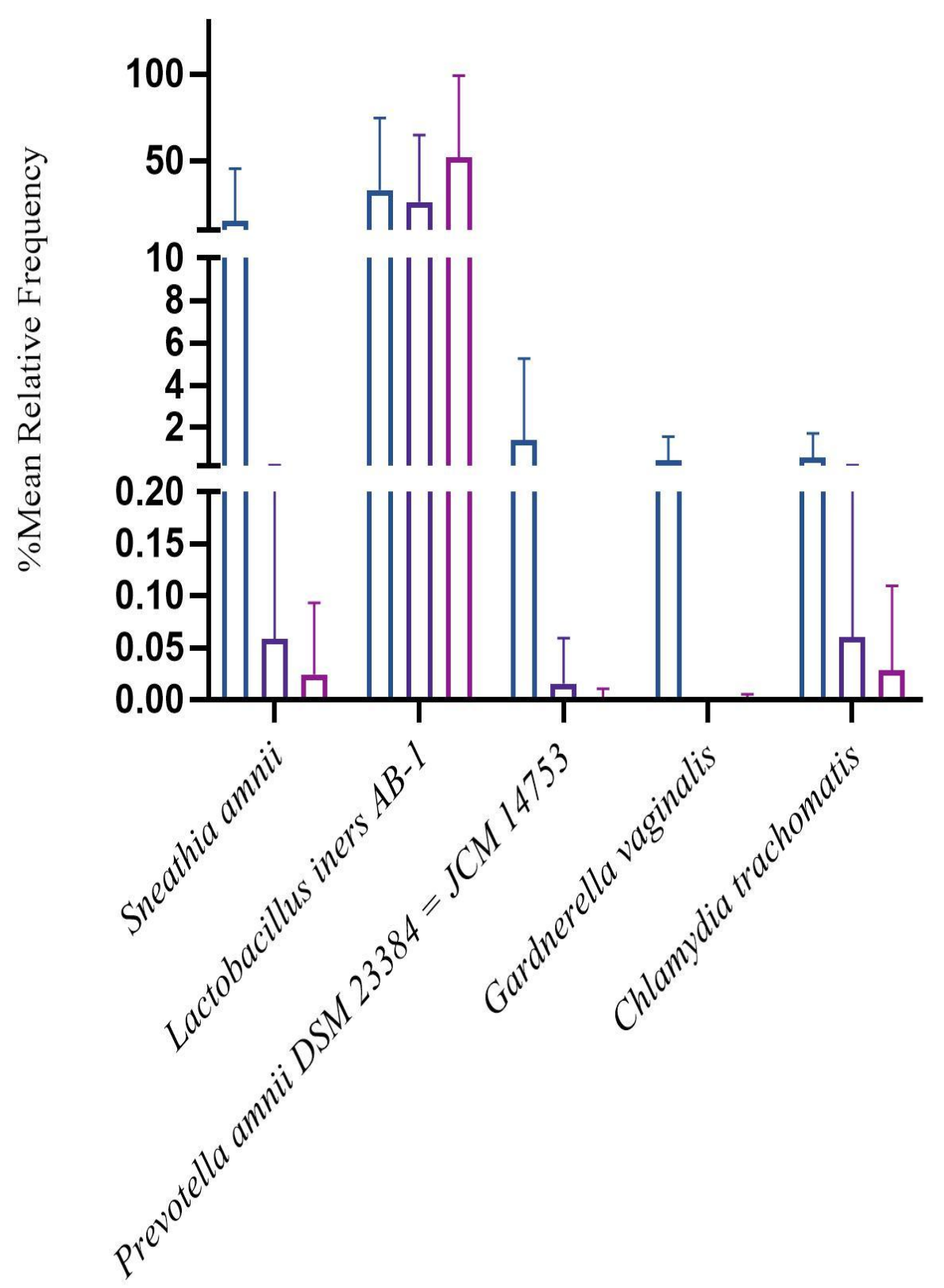

$\square$ CONTROL: mean rel. freq. (\%)

$\square$ RIF: mean rel. freq. (\%)

$\square$ UE: mean rel. freq. (\%) 


\section{Figure 6}

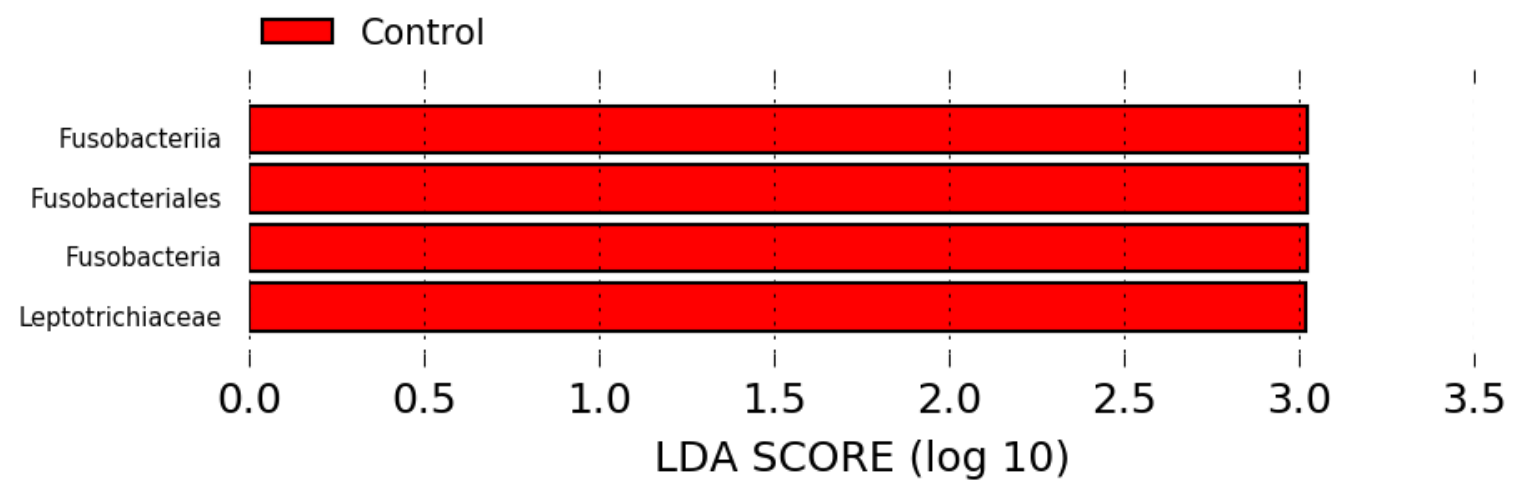


Figure 7

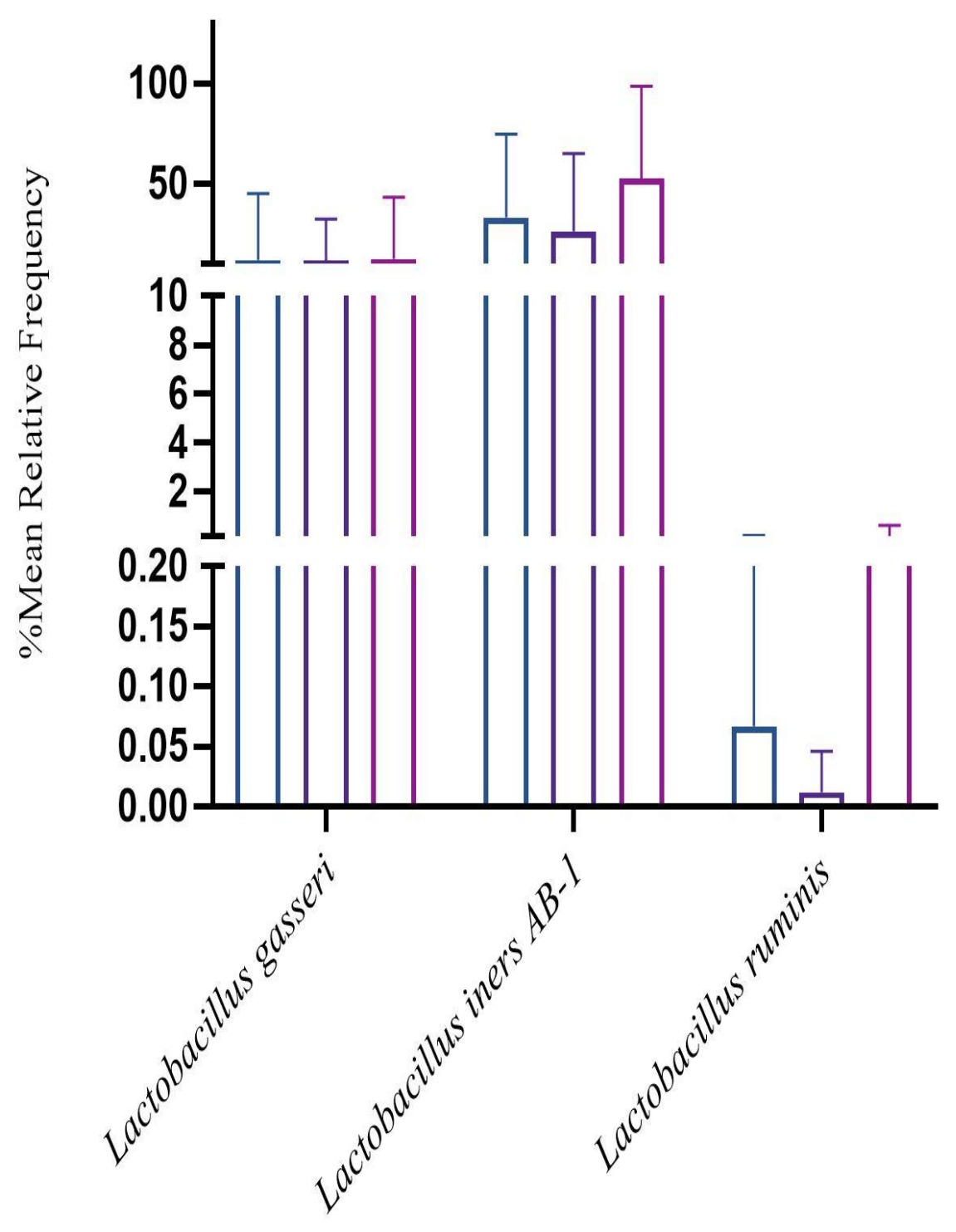

$\square$ CONTROL: mean rel. freq. ( $\%)$

$\square$ RIF: mean rel. freq. (\%)

$\square$ UE: mean rel. freq. (\%) 


\section{Supplementary figures}


Figure $1 S(a)$

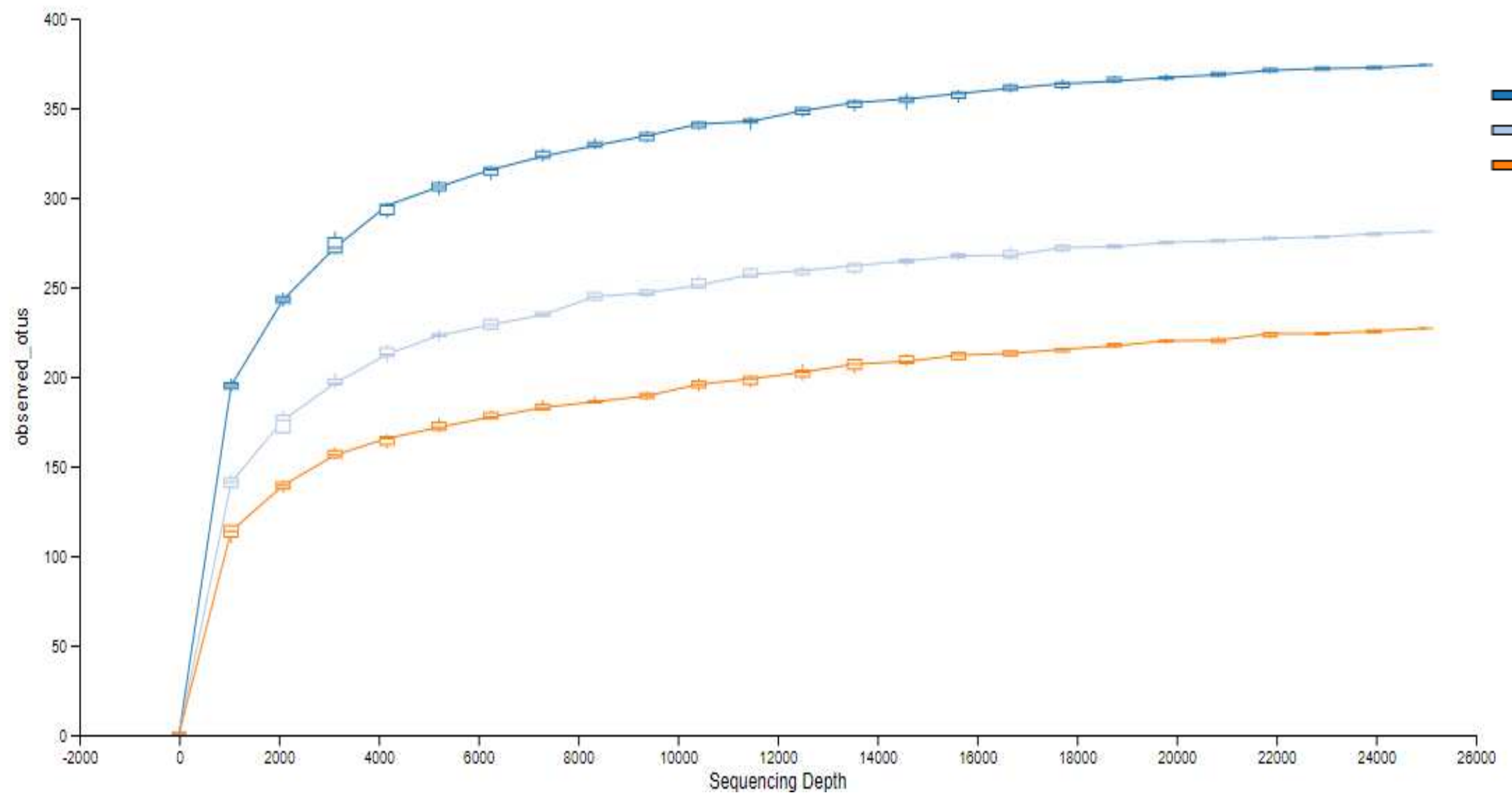


Figure $1 S(b)$

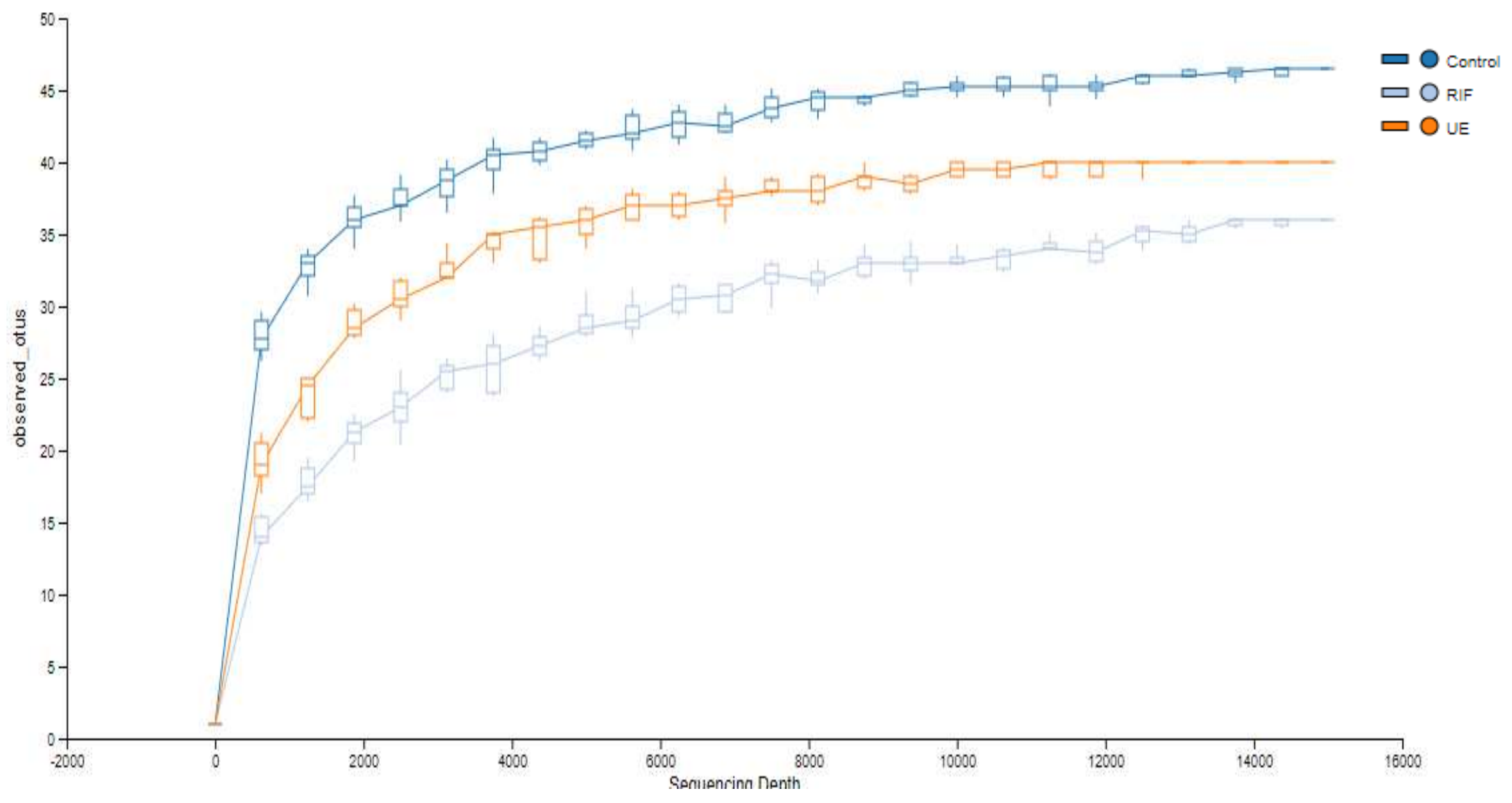


Figure $2 S(b)$

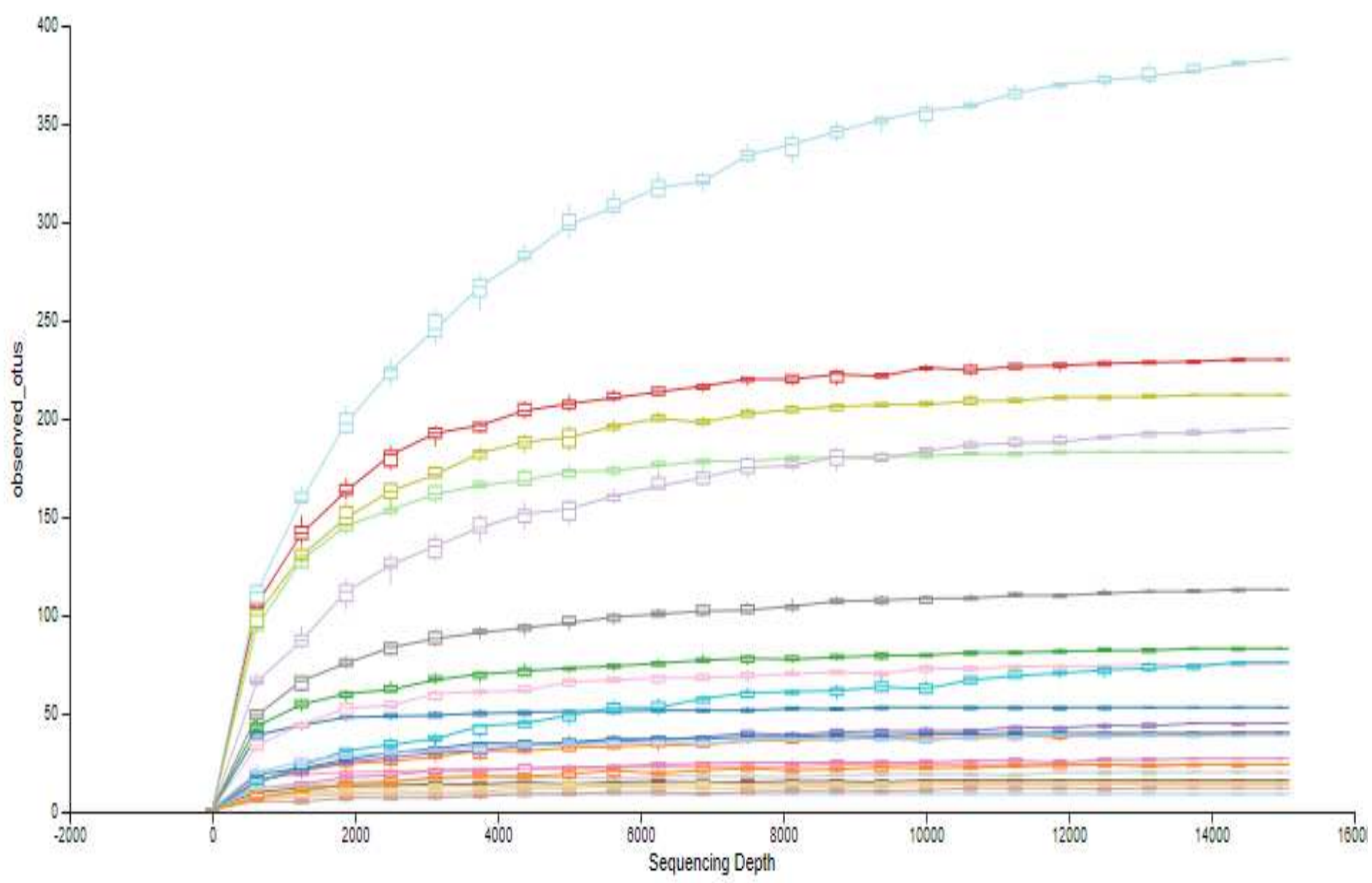

- $\mathrm{OCl}_{1}$

$\square \mathrm{Oc3}$

- $\mathrm{OCA}_{\mathrm{C}}$

口 O C5

D $\mathrm{O}$ c8

$\square \mathrm{Oc7}$

- $\mathrm{Oc8}$

$\square \mathrm{Ocg}$

- O RIF1

口 O RIF10

D O RIF2

ㅁ O RIF4

口 O RIF5

口 O RIF6

ㅁ O RIF7

口 O RIF9

口 O UE2

$\square$ O UE4

- O Ue5

$\square$ O UeB

- O ve7

$\square$ O UE8

口 O UE: 
Figure 35

Kruskal-Wallis, $\mathrm{P}=0.150 ; \mathrm{H}=2.06$

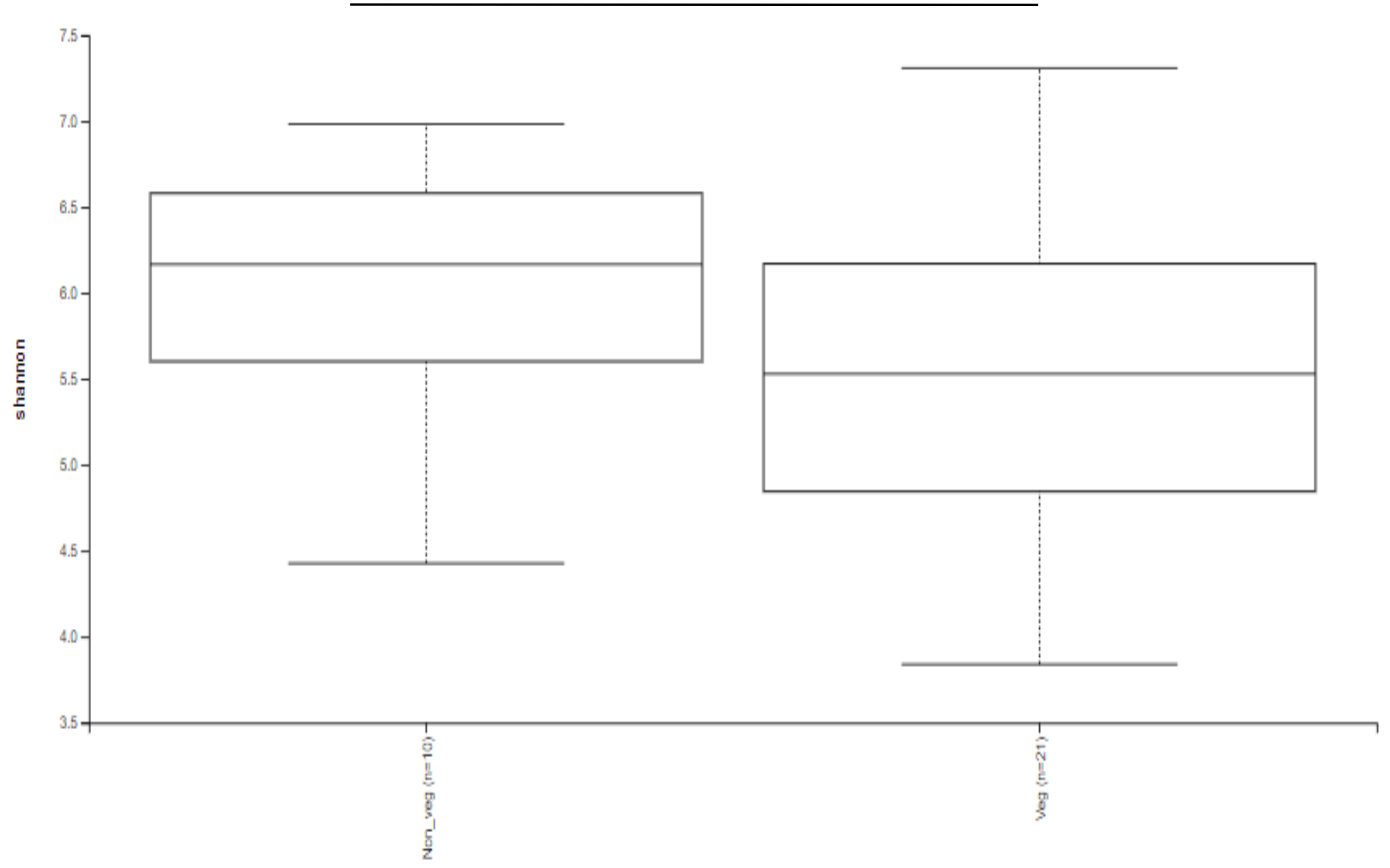


Figure 4S

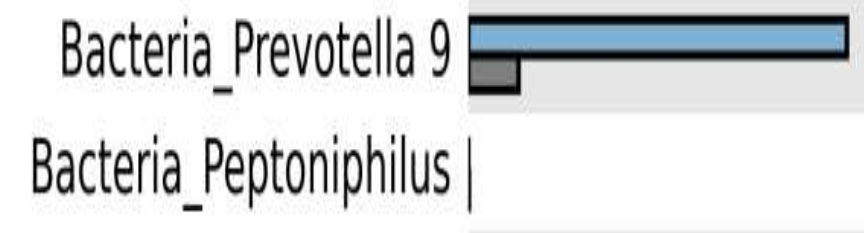

Bacteria_Ruminococcaceae UCG-004 $\mid$

$$
\text { Bacteria_Bacteroides }
$$

Bacteria_Ruminococcaceae UCG-010 P

$$
\text { Bacteria_Dorea — }
$$

Bacteria_TM7 phylum sp. oral clone FRO58 |

\section{5\% confidence intervals}

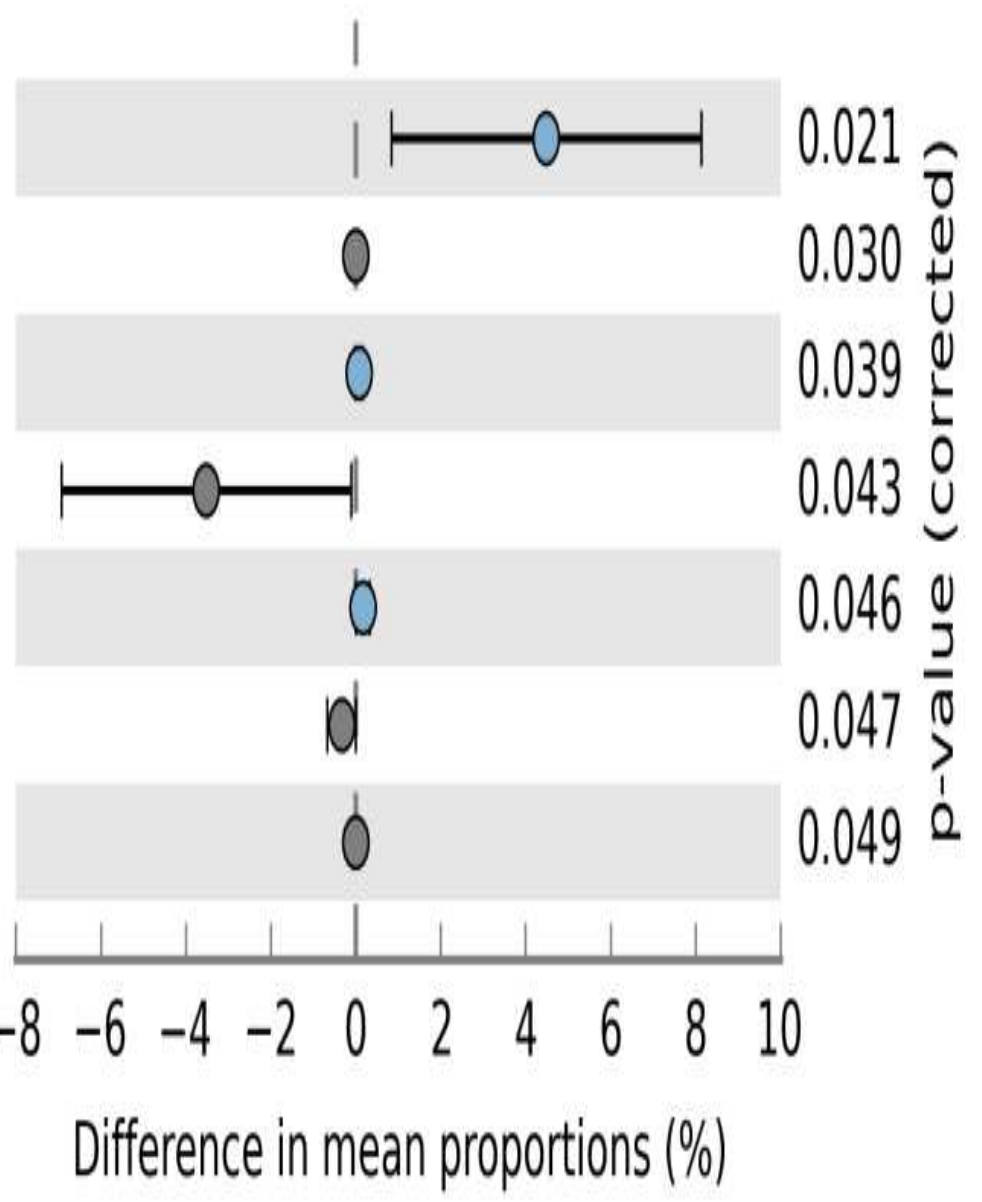

